\title{
A general reinforcement learning algorithm that masters chess, shogi and Go through self-play
}

\author{
David Silver, ${ }^{1,2 *}$ Thomas Hubert, ${ }^{1 *}$ Julian Schrittwieser, ${ }^{1 *}$ \\ Ioannis Antonoglou, ${ }^{1,2}$ Matthew Lai, ${ }^{1}$ Arthur Guez, ${ }^{1}$ Marc Lanctot, ${ }^{1}$ \\ Laurent Sifre, ${ }^{1}$ Dharshan Kumaran, ${ }^{1,2}$ Thore Graepel, ${ }^{1,2}$ \\ Timothy Lillicrap, ${ }^{1}$ Karen Simonyan, ${ }^{1}$ Demis Hassabis ${ }^{1}$ \\ ${ }^{1}$ DeepMind, 6 Pancras Square, London N1C 4AG. \\ ${ }^{2}$ University College London, Gower Street, London WC1E 6BT. \\ *These authors contributed equally to this work.
}

\begin{abstract}
The game of chess is the longest-studied domain in the history of artificial intelligence. The strongest programs are based on a combination of sophisticated search techniques, domain-specific adaptations, and handcrafted evaluation functions that have been refined by human experts over several decades. By contrast, the AlphaGo Zero program recently achieved superhuman performance in the game of Go by reinforcement learning from selfplay. In this paper, we generalize this approach into a single AlphaZero algorithm that can achieve superhuman performance in many challenging games. Starting from random play and given no domain knowledge except the game rules, AlphaZero convincingly defeated a world champion program in the games of chess and shogi (Japanese chess) as well as Go.
\end{abstract}

The study of computer chess is as old as computer science itself. Charles Babbage, Alan Turing, Claude Shannon, and John von Neumann devised hardware, algorithms and theory to analyse and play the game of chess. Chess subsequently became a grand challenge task for a generation of artificial intelligence researchers, culminating in high-performance computer chess programs that play at a super-human level $(1,2)$. However, these systems are highly tuned to their domain, and cannot be generalized to other games without substantial human effort, whereas general game-playing systems $(3,4)$ remain comparatively weak.

A long-standing ambition of artificial intelligence has been to create programs that can instead learn for themselves from first principles $(5,6)$. Recently, the AlphaGo Zero algorithm achieved superhuman performance in the game of Go, by representing Go knowledge using deep convolutional neural networks $(7,8)$, trained solely by reinforcement learning from games 
of self-play (9). In this paper, we introduce AlphaZero: a more generic version of the AlphaGo Zero algorithm that accomodates, without special-casing, to a broader class of game rules. We apply AlphaZero to the games of chess and shogi as well as Go, using the same algorithm and network architecture for all three games. Our results demonstrate that a general-purpose reinforcement learning algorithm can learn, tabula rasa - without domain-specific human knowledge or data, as evidenced by the same algorithm succeeding in multiple domains - superhuman performance across multiple challenging games.

A landmark for artificial intelligence was achieved in 1997 when Deep Blue defeated the human world chess champion (1). Computer chess programs continued to progress steadily beyond human level in the following two decades. These programs evaluate positions using handcrafted features and carefully tuned weights, constructed by strong human players and programmers, combined with a high-performance alpha-beta search that expands a vast search tree using a large number of clever heuristics and domain-specific adaptations. In (10) we describe these augmentations, focusing on the 2016 Top Chess Engine Championship (TCEC) season 9 world-champion Stockfish (11); other strong chess programs, including Deep Blue, use very similar architectures $(1,12)$.

In terms of game tree complexity, shogi is a substantially harder game than chess $(13,14)$ : it is played on a larger board with a wider variety of pieces; any captured opponent piece switches sides and may subsequently be dropped anywhere on the board. The strongest shogi programs, such as the 2017 Computer Shogi Association (CSA) world-champion Elmo, have only recently defeated human champions (15). These programs use an algorithm similar to those used by computer chess programs, again based on a highly optimized alpha-beta search engine with many domain-specific adaptations.

AlphaZero replaces the handcrafted knowledge and domain-specific augmentations used in traditional game-playing programs with deep neural networks, a general-purpose reinforcement learning algorithm, and a general-purpose tree search algorithm.

Instead of a handcrafted evaluation function and move ordering heuristics, AlphaZero uses a deep neural network $(\mathbf{p}, v)=f_{\theta}(s)$ with parameters $\theta$. This neural network $f_{\theta}(s)$ takes the board position $s$ as an input and outputs a vector of move probabilities $\mathbf{p}$ with components $p_{a}=\operatorname{Pr}(a \mid s)$ for each action $a$, and a scalar value $v$ estimating the expected outcome $z$ of the game from position $s, v \approx \mathbb{E}[z \mid s]$. AlphaZero learns these move probabilities and value estimates entirely from self-play; these are then used to guide its search in future games.

Instead of an alpha-beta search with domain-specific enhancements, AlphaZero uses a generalpurpose Monte Carlo tree search (MCTS) algorithm. Each search consists of a series of simulated games of self-play that traverse a tree from root state $s_{\text {root }}$ until a leaf state is reached. Each simulation proceeds by selecting in each state $s$ a move $a$ with low visit count (not previously frequently explored), high move probability and high value (averaged over the leaf states of simulations that selected $a$ from $s$ ) according to the current neural network $f_{\theta}$. The search returns a vector $\pi$ representing a probability distribution over moves, $\pi_{a}=\operatorname{Pr}\left(a \mid s_{\text {root }}\right)$.

The parameters $\theta$ of the deep neural network in AlphaZero are trained by reinforcement learning from self-play games, starting from randomly initialized parameters $\theta$. Each game is 
played by running an MCTS search from the current position $s_{\text {root }}=s_{t}$ at turn $t$, and then selecting a move, $a_{t} \sim \pi_{t}$, either proportionally (for exploration) or greedily (for exploitation) with respect to the visit counts at the root state. At the end of the game, the terminal position $s_{T}$ is scored according to the rules of the game to compute the game outcome $z:-1$ for a loss, 0 for a draw, and +1 for a win. The neural network parameters $\theta$ are updated to minimize the error between the predicted outcome $v_{t}$ and the game outcome $z$, and to maximize the similarity of the policy vector $\mathbf{p}_{t}$ to the search probabilities $\boldsymbol{\pi}_{t}$. Specifically, the parameters $\theta$ are adjusted by gradient descent on a loss function $l$ that sums over mean-squared error and cross-entropy losses,

$$
(\mathbf{p}, v)=f_{\theta}(s), \quad l=(z-v)^{2}-\boldsymbol{\pi}^{\top} \log \mathbf{p}+c\|\theta\|^{2},
$$

where $c$ is a parameter controlling the level of $L_{2}$ weight regularization. The updated parameters are used in subsequent games of self-play.

The AlphaZero algorithm described in this paper (see (10) for pseudocode) differs from the original AlphaGo Zero algorithm in several respects.

AlphaGo Zero estimated and optimized the probability of winning, exploiting the fact that Go games have a binary win or loss outcome. However, both chess and shogi may end in drawn outcomes; it is believed that the optimal solution to chess is a draw (16-18). AlphaZero instead estimates and optimizes the expected outcome.

The rules of Go are invariant to rotation and reflection. This fact was exploited in AlphaGo and AlphaGo Zero in two ways. First, training data were augmented by generating eight symmetries for each position. Second, during MCTS, board positions were transformed by using a randomly selected rotation or reflection before being evaluated by the neural network, so that the Monte Carlo evaluation was averaged over different biases. To accommodate a broader class of games, AlphaZero does not assume symmetry; the rules of chess and shogi are asymmetric (e.g. pawns only move forward, and castling is different on kingside and queenside). AlphaZero does not augment the training data and does not transform the board position during MCTS.

In AlphaGo Zero, self-play games were generated by the best player from all previous iterations. After each iteration of training, the performance of the new player was measured against the best player; if the new player won by a margin of $55 \%$ then it replaced the best player. By contrast, AlphaZero simply maintains a single neural network that is updated continually, rather than waiting for an iteration to complete. Self-play games are always generated by using the latest parameters for this neural network.

Like AlphaGo Zero, the board state is encoded by spatial planes based only on the basic rules for each game. The actions are encoded by either spatial planes or a flat vector, again based only on the basic rules for each game (10).

AlphaGo Zero used a convolutional neural network architecture that is particularly wellsuited to Go: the rules of the game are translationally invariant (matching the weight sharing structure of convolutional networks) and are defined in terms of liberties corresponding to the adjacencies between points on the board (matching the local structure of convolutional networks). By contrast, the rules of chess and shogi are position-dependent (e.g. pawns may 
A

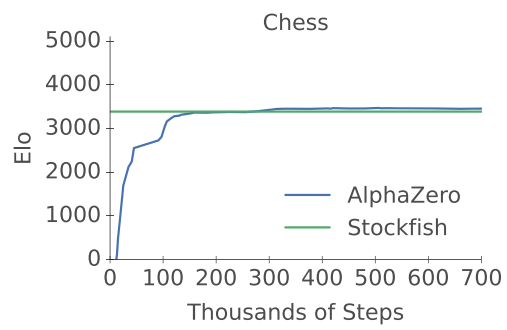

B

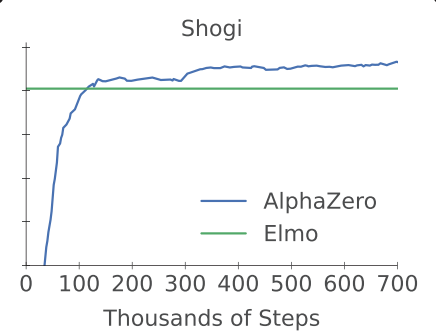

C

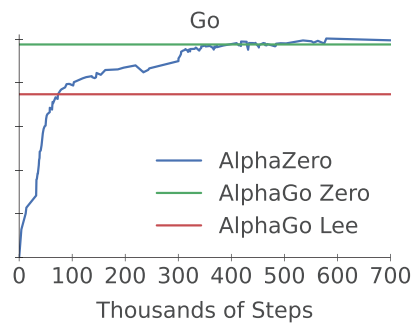

Figure 1: Training AlphaZero for 700,000 steps. Elo ratings were computed from games between different players where each player was given one second per move. (A) Performance of AlphaZero in chess, compared with the 2016 TCEC world-champion program Stockfish. (B) Performance of AlphaZero in shogi, compared with the 2017 CSA world-champion program Elmo. (C) Performance of AlphaZero in Go, compared with AlphaGo Lee and AlphaGo Zero (20 blocks over 3 days).

move two steps forward from the second rank and promote on the eighth rank) and include long-range interactions (e.g. the queen may traverse the board in one move). Despite these differences, AlphaZero uses the same convolutional network architecture as AlphaGo Zero for chess, shogi and Go.

The hyperparameters of AlphaGo Zero were tuned by Bayesian optimization. In AlphaZero we reuse the same hyperparameters, algorithm settings and network architecture for all games without game-specific tuning. The only exceptions are the exploration noise and the learning rate schedule (see (10) for further details).

We trained separate instances of AlphaZero for chess, shogi and Go. Training proceeded for 700,000 steps (in mini-batches of 4,096 training positions) starting from randomly initialized parameters. During training only, 5,000 first-generation tensor processing units (TPUs) (19) were used to generate self-play games, and 16 second-generation TPUs were used to train the neural networks. Training lasted for approximately 9 hours in chess, 12 hours in shogi and 13 days in Go (see table S3) (20). Further details of the training procedure are provided in (10).

Figure 1 shows the performance of AlphaZero during self-play reinforcement learning, as a function of training steps, on an Elo (21) scale (22). In chess, AlphaZero first outperformed Stockfish after just 4 hours (300,000 steps); in shogi, AlphaZero first outperformed Elmo after 2 hours (110,000 steps); and in Go, AlphaZero first outperformed AlphaGo Lee (9) after 30 hours $(74,000$ steps). The training algorithm achieved similar performance in all independent runs (see fig. S3), suggesting that the high performance of AlphaZero's training algorithm is repeatable.

We evaluated the fully trained instances of AlphaZero against Stockfish, Elmo and the previous version of AlphaGo Zero in chess, shogi and Go respectively. Each program was run on the hardware for which it was designed (23): Stockfish and Elmo used 44 central processing unit (CPU) cores (as in the TCEC world championship), whereas AlphaZero and AlphaGo Zero used a single machine with four first-generation TPUs and 44 CPU cores (24). The chess match 
was played against the 2016 TCEC (season 9) world champion Stockfish (see (10) for details). The shogi match was played against the 2017 CSA world champion version of Elmo (10). The Go match was played against the previously published version of AlphaGo Zero (also trained for 700,000 steps (25)). All matches were played using time controls of 3 hours per game, plus an additional 15 seconds for each move.

In Go, AlphaZero defeated AlphaGo Zero (9), winning 61\% of games. This demonstrates that a general approach can recover the performance of an algorithm that exploited board symmetries to generate eight times as much data (see also fig. $\mathbf{S 1}$.

In chess, AlphaZero defeated Stockfish, winning 155 games and losing 6 games out of 1,000 (Fig. 2). To verify the robustness of AlphaZero, we played additional matches that started from common human openings (Fig. 3). AlphaZero defeated Stockfish in each opening, suggesting that AlphaZero has mastered a wide spectrum of chess play. The frequency plots in Fig. 3 and the timeline in fig. $\mathrm{S} 2$ show that common human openings were independently discovered and played frequently by AlphaZero during self-play training. We also played a match that started from the set of opening positions used in the 2016 TCEC world championship; AlphaZero won convincingly in this match too (26) (see fig. S4). We played additional matches against the most recent development version of Stockfish (27), and a variant of Stockfish that uses a strong opening book (28). AlphaZero won all matches by a large margin (Fig. 2).

Table S6 shows 20 chess games played by AlphaZero in its matches against Stockfish. In several games AlphaZero sacrificed pieces for long-term strategic advantage, suggesting that it has a more fluid, context-dependent positional evaluation than the rule-based evaluations used by previous chess programs.

In shogi, AlphaZero defeated Elmo, winning 98.2\% of games when playing black, and $91.2 \%$ overall. We also played a match under the faster time controls used in the 2017 CSA world championship, and against another state-of-the-art shogi program (29); AlphaZero again won both matches by a wide margin (Fig. 2).

Table S7] shows 10 shogi games played by AlphaZero in its matches against Elmo. The frequency plots in Fig. 3 and the timeline in fig. $\mathrm{S} 2$ show that AlphaZero frequently plays one of the two most common human openings, but rarely plays the second, deviating on the very first move.

AlphaZero searches just 60,000 positions per second in chess and shogi, compared with 60 million for Stockfish and 25 million for Elmo (table S4). AlphaZero may compensate for the lower number of evaluations by using its deep neural network to focus much more selectively on the most promising variations (Fig. 4 provides an example from the match against Stockfish) - arguably a more "human-like" approach to search, as originally proposed by Shannon (30). AlphaZero also defeated Stockfish when given 1/10 as much thinking time as its opponent (i.e. searching 1/10,000 as many positions), and won $46 \%$ of games against Elmo when given $1 / 100$ as much time (i.e. searching $\sim 1 / 40,000$ as many positions), see Fig. 2. The high performance of AlphaZero, using MCTS, calls into question the widely held belief $(31,32)$ that alpha-beta search is inherently superior in these domains.

The game of chess represented the pinnacle of artificial intelligence research over several 
decades. State-of-the-art programs are based on powerful engines that search many millions of positions, leveraging handcrafted domain expertise and sophisticated domain adaptations. AlphaZero is a generic reinforcement learning and search algorithm - originally devised for the game of Go - that achieved superior results within a few hours, searching 1/1,000 as many positions, given no domain knowledge except the rules of chess. Furthermore, the same algorithm was applied without modification to the more challenging game of shogi, again outperforming state-of-the-art programs within a few hours. These results bring us a step closer to fulfilling a longstanding ambition of artificial intelligence (3): a general games playing system that can learn to master any game.

\section{References}

1. M. Campbell, A. J. Hoane, F. Hsu, Artificial Intelligence 134, 57 (2002).

2. F.-h. Hsu, Behind Deep Blue: Building the Computer that Defeated the World Chess Champion (Princeton University Press, 2002).

3. B. Pell, Computational Intelligence 12, 177 (1996).

4. M. R. Genesereth, N. Love, B. Pell, AI Magazine 26, 62 (2005).

5. A. L. Samuel, IBM Journal of Research and Development 11, 601 (1967).

6. G. Tesauro, Neural Computation 6, 215 (1994).

7. C. J. Maddison, A. Huang, I. Sutskever, D. Silver, International Conference on Learning Representations (2015).

8. D. Silver, et al., Nature 529, 484 (2016).

9. D. Silver, et al., Nature 550, 354 (2017).

10. See the supplementary materials for additional information.

11. T. Romstad, M. Costalba, J. Kiiski, et al., Stockfish: A strong open source chess engine. https://stockfishchess.org/, Retrieved November 29th, 2017.

12. D. N. L. Levy, M. Newborn, How Computers Play Chess (Ishi Press, 2009).

13. V. Allis, Searching for solutions in games and artificial intelligence, Ph.D. thesis, University of Limburg, Netherlands (1994).

14. H. Iida, M. Sakuta, J. Rollason, Artificial Intelligence 134, 121 (2002). 
A

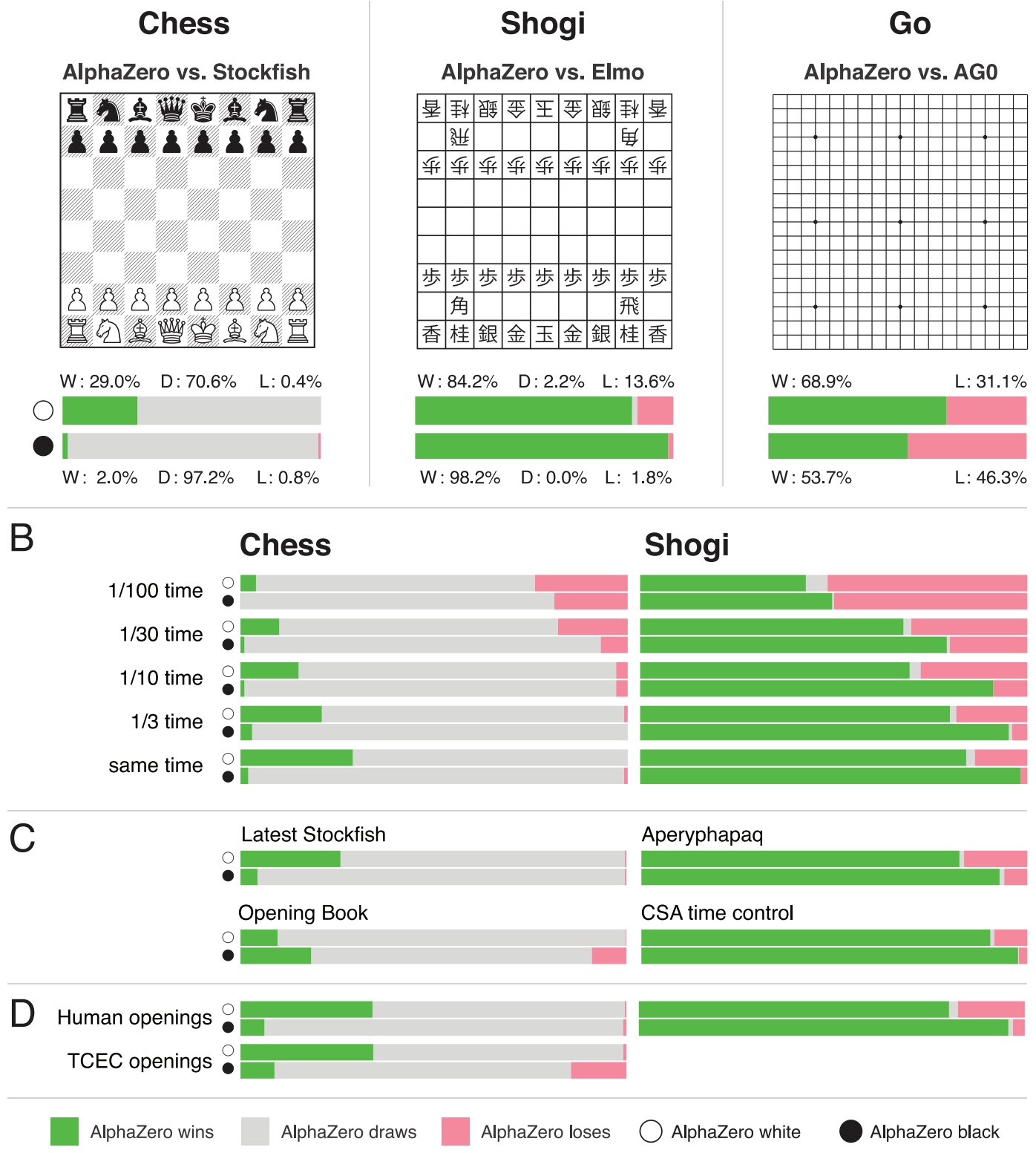

Figure 2: Comparison with specialized programs. (A) Tournament evaluation of AlphaZero in chess, shogi, and Go in matches against respectively Stockfish, Elmo, and the previously published version of AlphaGo Zero (AG0) that was trained for 3 days. In the top bar, AlphaZero plays white; in the bottom bar AlphaZero plays black. Each bar shows the results from AlphaZero's perspective: win ('W', green), draw ('D', grey), loss ('L', red). (B) Scalability of AlphaZero with thinking time, compared to Stockfish and Elmo. Stockfish and Elmo always receive full time ( 3 hours per game plus 15 seconds per move), time for AlphaZero is scaled down as indicated. (C) Extra evaluations of AlphaZero in chess against the most recent version of Stockfish at the time of writing (27), and against Stockfish with a strong opening book (28). Extra evaluations of AlphaZero in shogi were carried out against another strong shogi program Aperyqhapaq (29) at full time controls and against Elmo under 2017 CSA world championship time controls (10 minutes per game plus 10 seconds per move). (D) Average result of chess matches starting from different opening positions: either common human positions (see also Fig. 3), or the 2016 TCEC world championship opening positions (see also fig. S4). Average result of shogi matches starting from common human positions (see also Fig. 3). CSA world championship games start from the initial board position.

Match conditions are summarized in tables S8 and $\mathrm{S} 9$. 


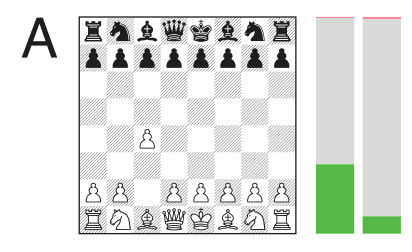

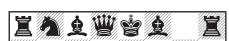

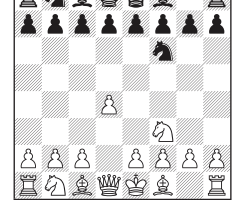

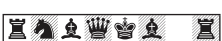

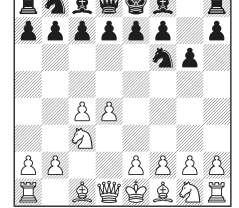

宣
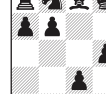

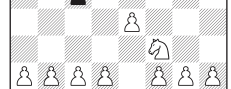

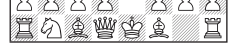

宣

i 1

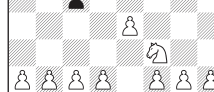

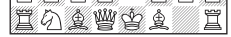

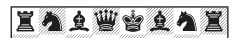

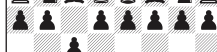

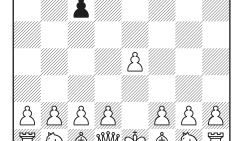

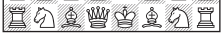
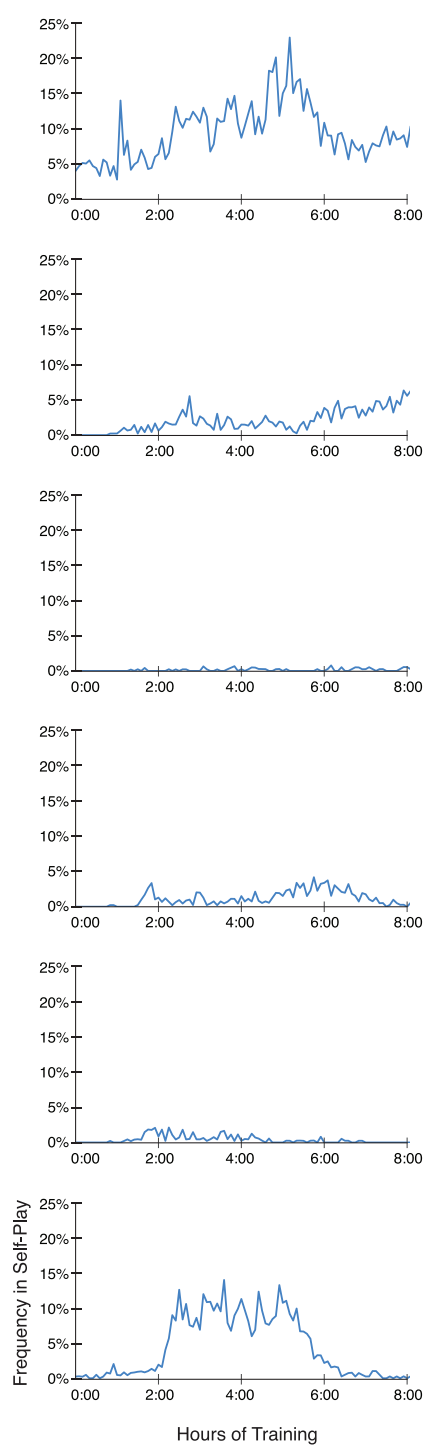

$\circ \bullet$
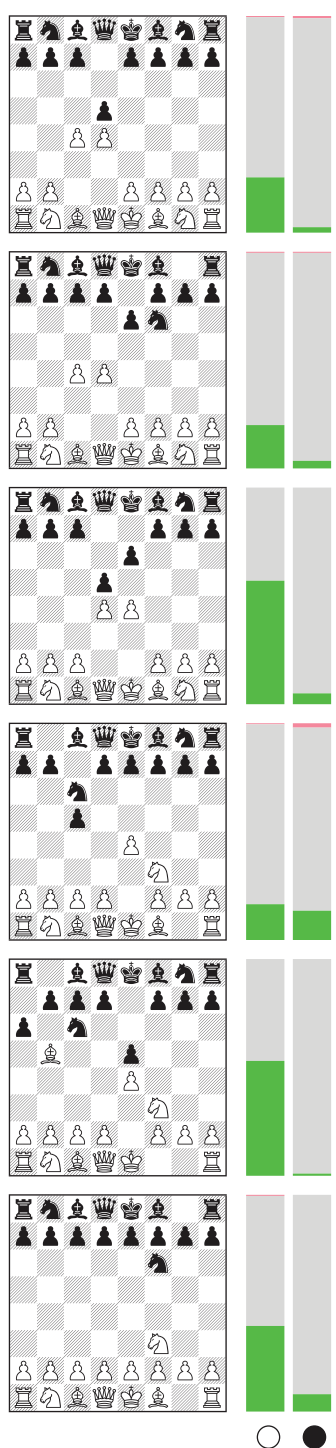
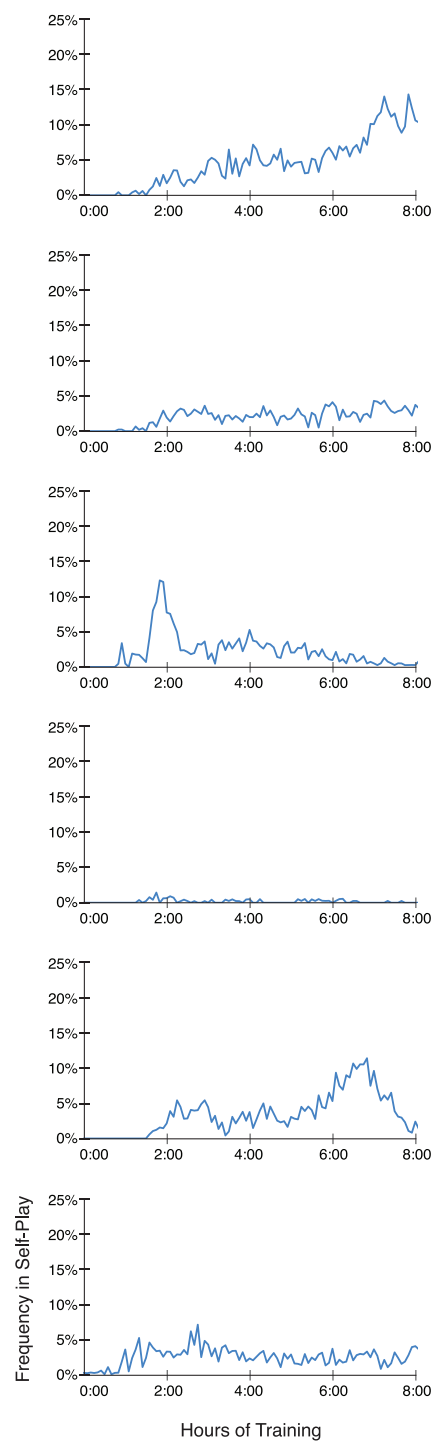

\section{B}
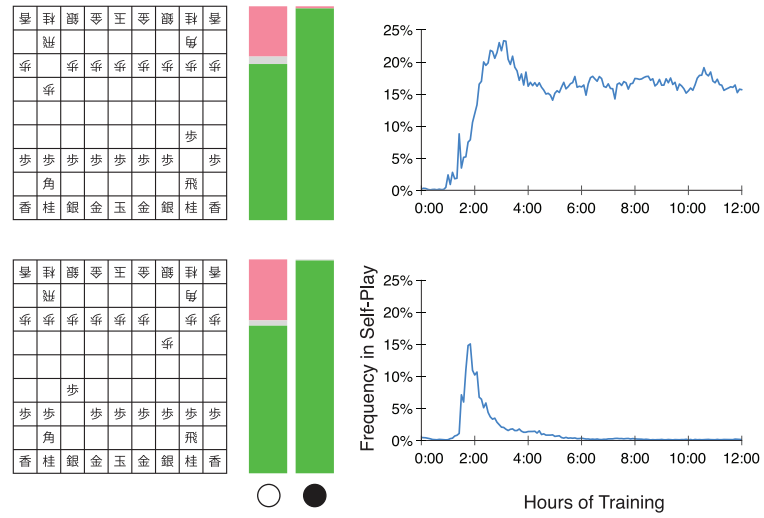
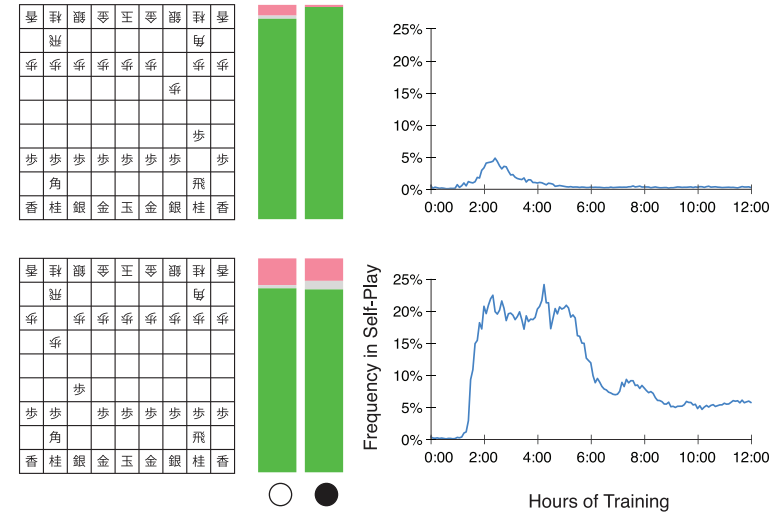

AlphaZero wins

AlphaZero draws

AlphaZero loses

AlphaZero white

AlphaZero black

Figure 3: Matches starting from the most popular human openings. AlphaZero plays against (A) Stockfish in chess and (B) Elmo in shogi. In the left bar, AlphaZero plays white, starting from the given position; in the right bar AlphaZero plays black. Each bar shows the results from AlphaZero's perspective: win (green), draw (grey), loss (red). The percentage frequency of self-play training games in which this opening was selected by AlphaZero is plotted against the duration of training, in hours. 

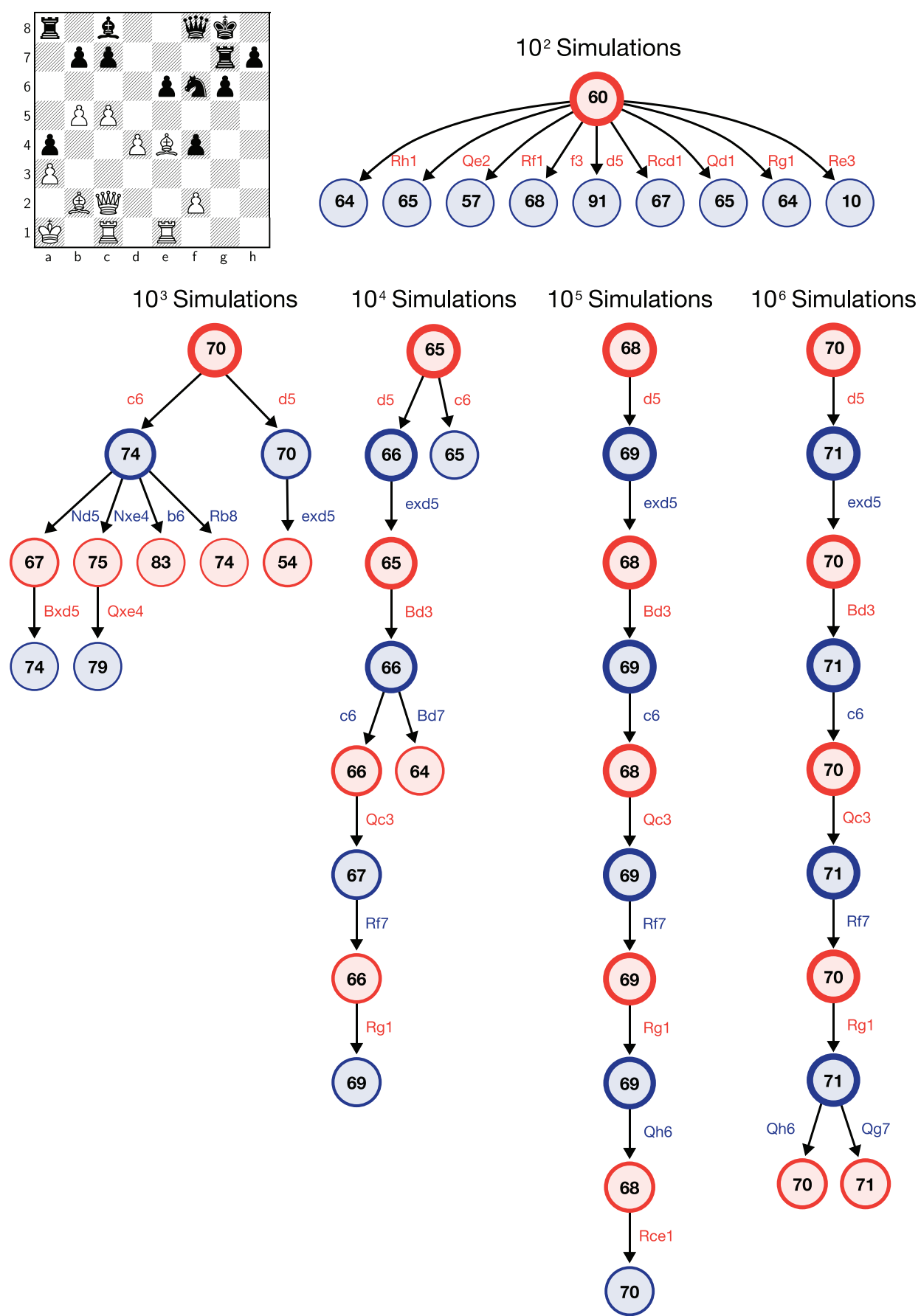

Figure 4: AlphaZero's search procedure. The search is illustrated for a position (inset) from game 1 (table S6) between AlphaZero (white) and Stockfish (black) after 29. ... Qf8. The internal state of AlphaZero's MCTS is summarized after $10^{2}, \ldots, 10^{6}$ simulations. Each summary shows the 10 most visited states. The estimated value is shown in each state, from white's perspective, scaled to the range $[0,100]$. The visit count of each state, relative to the root state of that tree, is proportional to the thickness of the border circle. AlphaZero considers 30. 6 but eventually plays $30 . \mathrm{d} 5$. 
15. C. S. Association, Results of the 27th world computer shogi championship. http: //www2.computer-shogi.org/wcsc27/index_e.html, Retrieved November 29 th, 2017.

16. W. Steinitz, The Modern Chess Instructor (Edition Olms AG, 1990).

17. E. Lasker, Common Sense in Chess (Dover Publications, 1965).

18. J. Knudsen, Essential Chess Quotations (iUniverse, 2000).

19. N. P. Jouppi, C. Young, N. Patil, et al., Proceedings of the 44th Annual International Symposium on Computer Architecture, ISCA '17 (ACM, 2017), pp. 1-12.

20. Note that the original AlphaGo Zero paper used GPUs to train the neural networks.

21. R. Coulom, International Conference on Computers and Games (2008), pp. 113-124.

22. The prevalence of draws in high-level chess tends to compress the Elo scale, compared to shogi or Go.

23. Stockfish is designed to exploit CPU hardware and cannot make use of GPU/TPU, whereas AlphaZero is designed to exploit GPU/TPU hardware rather than CPU.

24. A first generation TPU is roughly similar in inference speed to a Titan V GPU, although the architectures are not directly comparable.

25. AlphaGo Zero was ultimately trained for 3.1 million steps over 40 days.

26. Many TCEC opening positions are unbalanced according to both AlphaZero and Stockfish, resulting in more losses for both players.

27. Newest available version of Stockfish as of 13th of January 2018, from https://github.com/official-stockfish/Stockfish/commit/ b508f9561cc2302c129efe8d60f201ff03ee72c8.

28. Cerebellum opening book from https://zipproth.de/\#Brainfish_ download. AlphaZero did not use an opening book. To ensure diversity against a deterministic opening book, AlphaZero used a small amount of randomization in its opening moves (10); this avoided duplicate games but also resulted in more losses.

29. Aperyqhapaq's evaluation files are available at https://github.com/qhapaq-49/ qhapaq-bin/releases/tag/eloqhappa.

30. C. E. Shannon, The London, Edinburgh, and Dublin Philosophical Magazine and Journal of Science 41, 256 (1950). 
31. O. Arenz, Monte Carlo chess, Master's thesis, Technische Universitat Darmstadt (2012).

32. O. E. David, N. S. Netanyahu, L. Wolf, International Conference on Artificial Neural Networks (Springer, 2016), pp. 88-96.

\section{Supplemental References}

33. T. Marsland, Encyclopedia of Artificial Intelligence, S. Shapiro, ed. (John Wiley \& sons, New York, 1987).

34. G. Tesauro, Artificial Intelligence 134, 181 (2002).

35. G. Tesauro, G. R. Galperin, Advances in Neural Information Processing Systems 9 (1996), pp. $1068-1074$.

36. S. Thrun, Advances in Neural Information Processing Systems (1995), pp. 1069-1076.

37. D. F. Beal, M. C. Smith, Information Sciences 122, 3 (2000).

38. D. F. Beal, M. C. Smith, Theoretical Computer Science 252, 105 (2001).

39. J. Baxter, A. Tridgell, L. Weaver, Machine Learning 40, 243 (2000).

40. J. Veness, D. Silver, A. Blair, W. Uther, Advances in Neural Information Processing Systems (2009), pp. 1937-1945.

41. T. Kaneko, K. Hoki, Advances in Computer Games - 13th International Conference, ACG 2011, Tilburg, The Netherlands, November 20-22, 2011, Revised Selected Papers (2011), pp. 158-169.

42. K. Hoki, T. Kaneko, Journal of Artificial Intelligence Research (JAIR) 49, 527 (2014).

43. M. Lai, Giraffe: Using deep reinforcement learning to play chess, Master's thesis, Imperial College London (2015).

44. T. Anthony, Z. Tian, D. Barber, Advances in Neural Information Processing Systems 30 (2017), pp. 5366-5376.

45. D. E. Knuth, R. W. Moore, Artificial Intelligence 6, 293- (1975).

46. R. Ramanujan, A. Sabharwal, B. Selman, Proceedings of the 26th Conference on Uncertainty in Artificial Intelligence (UAI) (2010).

47. C. D. Rosin, Annals of Mathematics and Artificial Intelligence 61, 203 (2011). 
48. K. He, X. Zhang, S. Ren, J. Sun, 14th European Conference on Computer Vision (2016), pp. 630-645.

49. The TCEC world championship disallows opening books and instead starts two games (one from each colour) from each opening position.

50. Online chess games database, 365chess (2017). URL: https://www. 365chess . $\mathrm{com} /$. 


\section{Acknowledgments}

We thank Matthew Sadler for analysing chess games; Yoshiharu Habu for analysing shogi games; Lorrayne Bennett for organizational assistance; Bernhard Konrad, Ed Lockhart and Georg Ostrovski for reviewing the paper; and the rest of the DeepMind team for their support.

\section{Funding}

All research described in this report was funded by DeepMind and Alphabet.

\section{Author contributions}

D.S., J.S., T.H. and I.A. designed the AlphaZero algorithm with advice from T.G., A.G., T.L., K.S., M.Lai, L.S., M.Lanctot; J.S., I.A., T.H. and M.Lai implemented the AlphaZero program; T.H., J.S., D.S., M.Lai, I.A., T.G., K.S., D.K. and D.H. ran experiments and/or analysed data; D.S., T.H., J.S., and D.H. managed the project. D.S., J.S., T.H., M.Lai, I.A. and D.H., wrote the paper.

\section{Competing interests}

The authors declare no competing financial interests. DeepMind has filed the following patent applications related to this work: PCT/EP2018/063869; US15/280,711; US15/280,784.

\section{Data and materials availability}

A full description of the algorithm in pseudocode as well as additional games between AlphaZero and other programs are available in the Supplementary Materials.

\section{Supplementary Materials}

- 110 chess games between AlphaZero and Stockfish 8 from the initial board position.

- 100 chess games between AlphaZero and Stockfish 8 from 2016 TCEC start positions.

- 100 shogi games between AlphaZero and Elmo from the initial board position.

- Pseudocode description of the AlphaZero algorithm.

- Data for Figures 1 and 3 in JSON format.

- Supplementary Figures S1, S2, S3, S4 and Supplementary Tables S1, S2, S3, S4, S5, S6, S7, S8, S9.

- References (33-50). 


\section{Methods}

\section{Anatomy of a Computer Chess Program}

In this section we describe the components of a typical computer chess program, focusing specifically on Stockfish (11), an open source program that won the TCEC (Season 9) computer chess world championship in 2016. For an overview of standard methods, see (33).

Each position $s$ is described by a sparse vector of handcrafted features $\phi(s)$, including midgame/endgame-specific material point values, material imbalance tables, piece-square tables, mobility and trapped pieces, pawn structure, king safety, outposts, bishop pair, and other miscellaneous evaluation patterns. Each feature $\phi_{i}$ is assigned, by a combination of manual and automatic tuning, a corresponding weight $w_{i}$ and the position is evaluated by a linear combination $v(s, w)=\phi(s)^{\top} w$. However, this raw evaluation is only considered accurate for positions that are "quiet", with no unresolved captures or checks. A domain-specialized quiescence search is used to resolve ongoing tactical situations before the evaluation function is applied.

The final evaluation of a position $s$ is computed by a minimax search that evaluates each leaf using a quiescence search. Alpha-beta pruning is used to safely cut any branch that is provably dominated by another variation. Additional cuts are achieved using aspiration windows and principal variation search. Other pruning strategies include null move pruning (which assumes a pass move should be worse than any variation, in positions that are unlikely to be in zugzwang, as determined by simple heuristics), futility pruning (which assumes knowledge of the maximum possible change in evaluation), and other domain-dependent pruning rules (which assume knowledge of the value of captured pieces).

The search is focused on promising variations both by extending the search depth of promising variations, and by reducing the search depth of unpromising variations based on heuristics like history, static-exchange evaluation (SEE), and moving piece type. Extensions are based on domain-independent rules that identify singular moves with no sensible alternative, and domaindependent rules, such as extending check moves. Reductions, such as late move reductions, are based heavily on domain knowledge.

The efficiency of alpha-beta search depends critically upon the order in which moves are considered. Moves are therefore ordered by iterative deepening (using a shallower search to order moves for a deeper search). In addition, a combination of domain-independent move ordering heuristics, such as killer heuristic, history heuristic, counter-move heuristic, and also domain-dependent knowledge based on captures (SEE) and potential captures (MVV/LVA).

A transposition table facilitates the reuse of values and move orders when the same position is reached by multiple paths. In some variants, a carefully tuned opening book may be used to select moves at the start of the game. An endgame tablebase, precalculated by exhaustive retrograde analysis of endgame positions, provides the optimal move in all positions with six and sometimes seven pieces or less.

Other strong chess programs, and also earlier programs such as Deep Blue (1), have used very similar architectures (33) including the majority of the components described above, al- 
though important details vary considerably.

None of the techniques described in this section are used by AlphaZero. It is likely that some of these techniques could further improve the performance of AlphaZero; however, we have focused on a pure self-play reinforcement learning approach and leave these extensions for future research.

\section{Prior Work on Computer Chess and Shogi}

In this section we discuss some notable prior work on reinforcement learning and/or deep learning in computer chess, shogi and, due to its historical relevance, backgammon.

TD Gammon (6) was a backgammon program that evaluated positions by a multi-layer perceptron, trained by temporal-difference learning to predict the final game outcome. When its evaluation function was combined with a 3-ply search (34) TD Gammon defeated the human world champion. A subsequent paper introduced the first version of Monte-Carlo search (35), which evaluated root positions by the average outcome of $n$-step rollouts. Each rollout was generated by greedy move selection and the $n$th position was evaluated by TD Gammon's neural network.

NeuroChess (36) evaluated positions by a neural network that used 175 handcrafted input features. It was trained by temporal-difference learning to predict the final game outcome, and also the expected features after two moves. NeuroChess won 13\% of games against GnuChess using a fixed depth 2 search, but lost overall.

Beal and Smith applied temporal-difference learning to estimate the piece values in chess (37) and shogi (38), starting from random values and learning solely by self-play.

KnightCap (39) evaluated positions by a neural network that used an attack table based on knowledge of which squares are attacked or defended by which pieces. It was trained by a variant of temporal-difference learning, known as TD(leaf), that updates the leaf value of the principal variation of an alpha-beta search. KnightCap achieved human master level after training against a strong computer opponent with hand-initialized piece-value weights.

Meep (40) evaluated positions by a linear evaluation function based on handcrafted features. It was trained by another variant of temporal-difference learning, known as TreeStrap, that updates all nodes of an alpha-beta search. Meep defeated human international master players in 13 out of 15 games, after training by self-play with randomly initialized weights.

Kaneko and Hoki (41) trained the weights of a shogi evaluation function comprising a million features, by learning to select expert human moves during alpha-beta search. They also performed a large-scale optimization based on minimax search regulated by expert game logs (42); this formed part of the Bonanza engine that won the 2013 World Computer Shogi Championship.

Giraffe (43) evaluated positions by a neural network that included mobility maps and attack and defend maps describing the lowest valued attacker and defender of each square. It was trained by self-play using TD(leaf), also reaching a standard of play comparable to international masters. 
DeepChess (32) trained a neural network to perform pair-wise evaluations of positions. It was trained by supervised learning from a database of human expert games that was pre-filtered to avoid capture moves and drawn games. DeepChess reached a strong grandmaster level of play.

All of these programs combined their learned evaluation functions with an alpha-beta search enhanced by a variety of extensions.

By contrast, an approach based on training dual policy and value networks using a policy iteration algorithm similar to AlphaZero was successfully applied to the game Hex (44). This work differed from AlphaZero in several regards: the policy network was initialized by imitating a pre-existing MCTS search algorithm, augmented by rollouts; the network was subsequently retrained from scratch at each iteration; and value targets were based on the outcome of self-play games using the raw policy network, rather than MCTS search.

\section{MCTS and Alpha-Beta Search}

For at least four decades the strongest computer chess programs have used alpha-beta search with handcrafted evaluation functions $(33,45)$. Chess programs using traditional MCTS (31) were much weaker than alpha-beta search programs (46), whereas alpha-beta programs based on neural networks have previously been unable to compete with faster, handcrafted evaluation functions. Surprisingly, AlphaZero surpassed previous approaches by using an effective combination of MCTS and neural networks.

AlphaZero evaluates positions non-linearly using deep neural networks, rather than the linear evaluation function used in typical chess programs. This provides a more powerful evaluation function, but may also introduce larger worst-case generalization errors. When combined with alpha-beta search, which computes an explicit minimax, the biggest errors are typically propagated directly to the root of the subtree. By contrast, AlphaZero's MCTS averages over the position evaluations within a subtree, rather than computing the minimax evaluation of that subtree. We speculate that the approximation errors introduced by neural networks therefore tend to cancel out when evaluating a large subtree.

\section{Domain Knowledge}

AlphaZero was provided with the following domain knowledge about each game:

1. The input features describing the position, and the output features describing the move, are structured as a set of planes; i.e. the neural network architecture is matched to the grid-structure of the board.

2. AlphaZero is provided with perfect knowledge of the game rules. These are used during MCTS, to simulate the positions resulting from a sequence of moves, to determine game termination, and to score any simulations that reach a terminal state. 
3. Knowledge of the rules is also used to encode the input planes (i.e. castling, repetition, no-progress) and output planes (how pieces move, promotions, and piece drops in shogi).

4. The typical number of legal moves is used to scale the exploration noise (see below).

5. Chess and shogi games exceeding 512 steps were terminated and assigned a drawn outcome; Go games exceeding 722 steps were terminated and scored with Tromp-Taylor rules, similarly to previous work (9).

AlphaZero did not use an opening book, endgame tablebases, or domain-specific heuristics.

\section{Search}

We briefly describe here the MCTS algorithm (9) used by AlphaZero; further details can be found in the pseudocode in the Supplementary Data. Each state-action pair $(s, a)$ stores a set of statistics, $\{N(s, a), W(s, a), Q(s, a), P(s, a)\}$, where $N(s, a)$ is the visit count, $W(s, a)$ is the total action-value, $Q(s, a)$ is the mean action-value, and $P(s, a)$ is the prior probability of selecting $a$ in $s$. Each simulation begins at the root node of the search tree, $s_{0}$, and finishes when the simulation reaches a leaf node $s_{L}$ at time-step $L$. At each of these timesteps, $t<L$, an action is selected, $a_{t}=\arg \max _{a}\left(Q\left(s_{t}, a\right)+U\left(s_{t}, a\right)\right)$, using a variant of the PUCT algorithm (47), $U(s, a)=C(s) P(s, a) \sqrt{N(s)} /(1+N(s, a))$, where $N(s)$ is the parent visit count and $C(s)$ is the exploration rate, which grows slowly with search time, $C(s)=\log \left(\left(1+N(s)+c_{\text {base }}\right) / c_{\text {base }}\right)+c_{\text {init }}$, but is essentially constant during the fast training games. The leaf node $s_{L}$ is added to a queue for neural network evaluation, $(\mathbf{p}, v)=f_{\theta}\left(s_{L}\right)$. The leaf node is expanded and each state-action pair $\left(s_{L}, a\right)$ is initialized to $\left\{N\left(s_{L}, a\right)=\right.$ $\left.0, W\left(s_{L}, a\right)=0, Q\left(s_{L}, a\right)=0, P\left(s_{L}, a\right)=p_{a}\right\}$. The visit counts and values are then updated in a backward pass through each step $t \leq L, N\left(s_{t}, a_{t}\right)=N\left(s_{t}, a_{t}\right)+1, W\left(s_{t}, a_{t}\right)=$ $W\left(s_{t}, a_{t}\right)+v, Q\left(s_{t}, a_{t}\right)=\frac{W\left(s_{t}, a_{t}\right)}{N\left(s_{t}, a_{t}\right)}$.

\section{Representation}

In this section we describe the representation of the board inputs, and the representation of the action outputs, used by the neural network in AlphaZero. Other representations could have been used; in our experiments the training algorithm worked robustly for many reasonable choices.

The input to the neural network is an $N \times N \times(M T+L)$ image stack that represents state using a concatenation of $T$ sets of $M$ planes of size $N \times N$. Each set of planes represents the board position at a time-step $t-T+1, \ldots, t$, and is set to zero for time-steps less than 1. The board is oriented to the perspective of the current player. The $M$ feature planes are composed of binary feature planes indicating the presence of the player's pieces, with one plane for each piece type, and a second set of planes indicating the presence of the opponent's pieces. For shogi there are additional planes indicating the number of captured prisoners of each type. There are an additional $L$ constant-valued input planes denoting the player's colour, the move 
number, and the state of special rules: the legality of castling in chess (kingside or queenside); the repetition count for the current position ( 3 repetitions is an automatic draw in chess; 4 in shogi); and the number of moves without progress in chess (50 moves without progress is an automatic draw). Input features are summarized in Table $\mathrm{S} 1$.

A move in chess may be described in two parts: first selecting the piece to move, and then selecting among possible moves for that piece. We represent the policy $\pi(a \mid s)$ by a $8 \times 8 \times 73$ stack of planes encoding a probability distribution over 4,672 possible moves. Each of the $8 \times 8$ positions identifies the square from which to "pick up" a piece. The first 56 planes encode possible 'queen moves' for any piece: a number of squares [1..7] in which the piece will be moved, along one of eight relative compass directions $\{N, N E, E, S E, S, S W, W, N W\}$. The next 8 planes encode possible knight moves for that piece. The final 9 planes encode possible underpromotions for pawn moves or captures in two possible diagonals, to knight, bishop or rook respectively. Other pawn moves or captures from the seventh rank are promoted to a queen.

The policy in shogi is represented by a $9 \times 9 \times 139$ stack of planes similarly encoding a probability distribution over 11,259 possible moves. The first 64 planes encode 'queen moves' and the next 2 planes encode knight moves. An additional $64+2$ planes encode promoting queen moves and promoting knight moves respectively. The last 7 planes encode a captured piece dropped back into the board at that location.

The policy in Go is represented identically to AlphaGo Zero (9), using a flat distribution over $19 \times 19+1$ moves representing possible stone placements and the pass move. We also tried using a flat distribution over moves for chess and shogi; the final result was almost identical although training was slightly slower.

Illegal moves are masked out by setting their probabilities to zero, and re-normalising the probabilities over the remaining set of legal moves.

The action representations are summarized in Table $\mathrm{S} 2$.

\section{Architecture}

Apart from the representation of positions and actions described above, AlphaZero uses the same network architecture as AlphaGo Zero (9), briefly recapitulated here.

The neural network consists of a "body" followed by both policy and value "heads". The body consists of a rectified batch-normalized convolutional layer followed by 19 residual blocks (48). Each such block consists of two rectified batch-normalized convolutional layers with a skip connection. Each convolution applies 256 filters of kernel size $3 \times 3$ with stride 1 . The policy head applies an additional rectified, batch-normalized convolutional layer, followed by a final convolution of 73 filters for chess or 139 filters for shogi, or a linear layer of size 362 for Go, representing the logits of the respective policies described above. The value head applies an additional rectified, batch-normalized convolution of 1 filter of kernel size $1 \times 1$ with stride 1 , followed by a rectified linear layer of size 256 and a tanh-linear layer of size 1 . 


\section{Configuration}

During training, each MCTS used 800 simulations. The number of games, positions, and thinking time varied per game due largely to different board sizes and game lengths, and are shown in Table S3. The learning rate was set to 0.2 for each game, and was dropped three times during the course of training to $0.02,0.002$ and 0.0002 respectively, after 100,300 and 500 thousands of steps for chess and shogi, and after 0, 300 and 500 thousands of steps for Go. Moves are selected in proportion to the root visit count. Dirichlet noise $\operatorname{Dir}(\alpha)$ was added to the prior probabilities in the root node; this was scaled in inverse proportion to the approximate number of legal moves in a typical position, to a value of $\alpha=\{0.3,0.15,0.03\}$ for chess, shogi and Go respectively. Positions were batched across parallel training games for evaluation by the neural network. Unless otherwise specified, the training and search algorithm and parameters are identical to AlphaGo Zero (9).

During evaluation, AlphaZero selects moves greedily with respect to the root visit count. Each MCTS was executed on a single machine with 4 first-generation TPUs.

\section{Opponents}

To evaluate performance in chess, we used Stockfish version 8 (official Linux release) as a baseline program. Stockfish was configured according to its 2016 TCEC world championship superfinal settings: 44 threads on 44 cores (two 2.2GHz Intel Xeon Broadwell CPUs with 22 cores), a hash size of 32GB, syzygy endgame tablebases, at 3 hour time controls with 15 additional seconds per move. We also evaluated against the most recent version, Stockfish 9 (just released at time of writing), using the same configuration.

Stockfish does not have an opening book of its own and all primary evaluations were performed without an opening book. We also performed one secondary evaluation in which the opponent's opening moves were selected by the Brainfish program, using an opening book derived from Stockfish. However, we note that these matches were low in diversity, and AlphaZero and Stockfish tended to produce very similar games throughout the match, more than $90 \%$ of which were draws. When we forced AlphaZero to play with greater diversity (by softmax sampling with a temperature of 10.0 among moves for which the value was no more than $1 \%$ away from the best move for the first 30 plies) the winning rate increased from $5.8 \%$ to $14 \%$.

To evaluate performance in shogi, we used Elmo version WCSC27 in combination with YaneuraOu 2017 Early KPPT 4.79 64AVX2 TOURNAMENT as a baseline program, using 44 CPU threads (on two 2.2GHz Intel Xeon Broadwell CPUs with 22 cores) and a hash size of 32GB with the usi options of EnteringKingRule set to CSARule27, MinimumThinkingTime set to 1000, BookFile set to standard_book.db, BookDepthLimit set to 0 and BookMoves set to 200. Additionally, we also evaluated against Aperyqhapaq combined with the same YaneuraOu

version and no book file. For Aperyqhapaq, we used the same usi options as for Elmo except for the book setting. 


\section{Match conditions}

We measured the head-to-head performance of AlphaZero in matches against each of the above opponents (Figure 2). Three types of match were played: starting from the initial board position (the default configuration, unless otherwise specified); starting from human opening positions; or starting from the 2016 TCEC opening positions (49).

The majority of matches for chess, shogi and Go used the 2016 TCEC superfinal time controls: 3 hours of main thinking time, plus 15 additional seconds of thinking time for each move. We also investigated asymmetric time controls (Figure 2B), where the opponent received 3 hours of main thinking time but AlphaZero received only a fraction of this time. Finally, for shogi only, we ran a match using faster time controls used in the 2017 CSA world championship: 10 minutes per game plus 10 seconds per move.

AlphaZero used a simple time control strategy: thinking for $1 / 20$ th of the remaining time. Opponent programs used customized, sophisticated heuristics for time control. Pondering was disabled for all players (particularly important for the asymmetric time controls in Figure 2).

Resignation was enabled for all players (-650 centipawns for 4 consecutive moves for Stockfish, $-4,500$ centipawns for 10 consecutive moves for Elmo, or a value of -0.9 for AlphaZero and AlphaGo Lee).

Matches consisted of 1,000 games, except for the human openings (200 games as black and 200 games as white from each opening) and the 2016 TCEC openings (50 games as black and 50 games as white from each of the 50 openings). The human opening positions were chosen as those played more than 100,000 times in an online database (50).

\section{Elo ratings}

We evaluated the relative strength of AlphaZero (Figure 1) by measuring the Elo rating of each player. We estimate the probability that player $a$ will defeat player $b$ by a logistic function $p(a$ defeats $b)=\left(1+10^{\left(c_{\text {elo }}(e(b)-e(a))\right)}\right)^{-1}$, and estimate the ratings $e(\cdot)$ by Bayesian logistic regression, computed by the BayesElo program (21) using the standard constant $c_{\text {elo }}=1 / 400$.

Elo ratings were computed from the results of a 1 second per move tournament between iterations of AlphaZero during training, and also a baseline player: either Stockfish, Elmo or AlphaGo Lee respectively. The Elo rating of the baseline players was anchored to publicly available values (9).

In order to compare Elo ratings at 1 second per move time controls to standard Elo ratings at full time controls, we also provide the results of Stockfish vs. Stockfish and Elmo vs. Elmo matches (Table S5).

\section{Example games}

The Supplementary Data includes 110 games from the main chess match between AlphaZero and Stockfish, starting from the initial board position; 100 games from the chess match starting 
from 2016 TCEC world championship opening positions; and 100 games from the main shogi match between AlphaZero and Elmo. For the chess match from the initial board position, one game was selected at random for each unique opening sequence of 30 plies; all AlphaZero losses were also included. For the TCEC match, one game as white and one game as black were selected at random from the match starting from each opening position. For the shogi match, one game was selected at random for each unique opening sequence of 25 plies (when AlphaZero was black) or 10 plies (when AlphaZero was white).

10 chess games were independently selected from each batch by GM Matthew Sadler, according to their interest to the chess community; these games are included in Table S6. Similarly, 10 shogi games were independently selected by Yoshiharu Habu; these games are included in Table $\mathbf{S 7}$ 

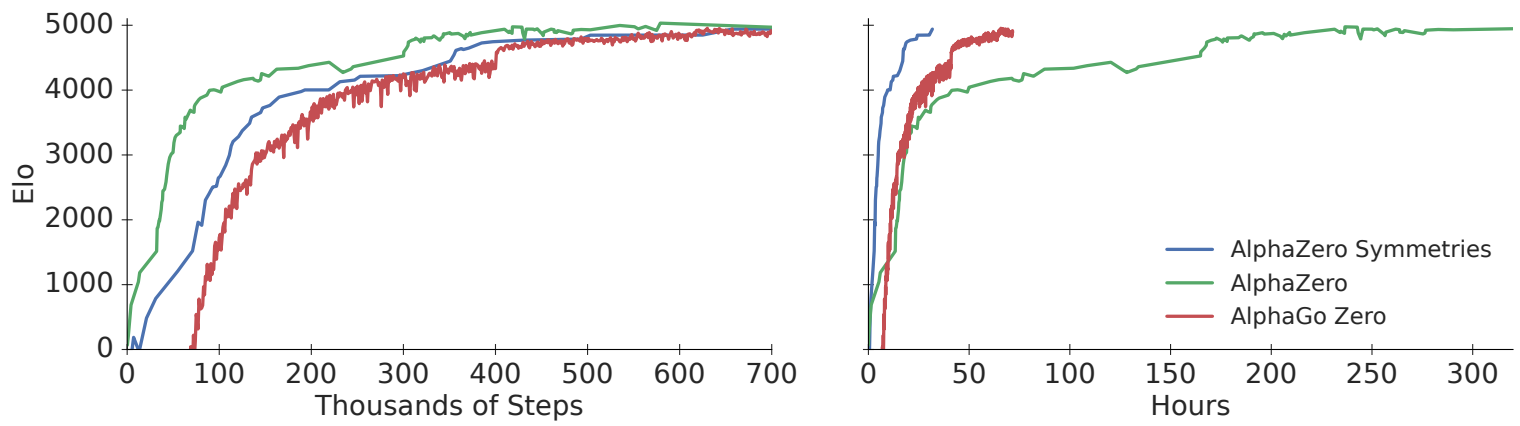

Figure S1: Learning curves showing the Elo performance during training in Go. Comparison between AlphaZero, a version of AlphaZero that exploits knowledge of symmetries in a similar manner to AlphaGo Zero, and the previously published AlphaGo Zero. AlphaZero generates approximately $1 / 8$ as many positions per training step, and therefore uses eight times more wall clock time, than the symmetry-augmented algorithms. 


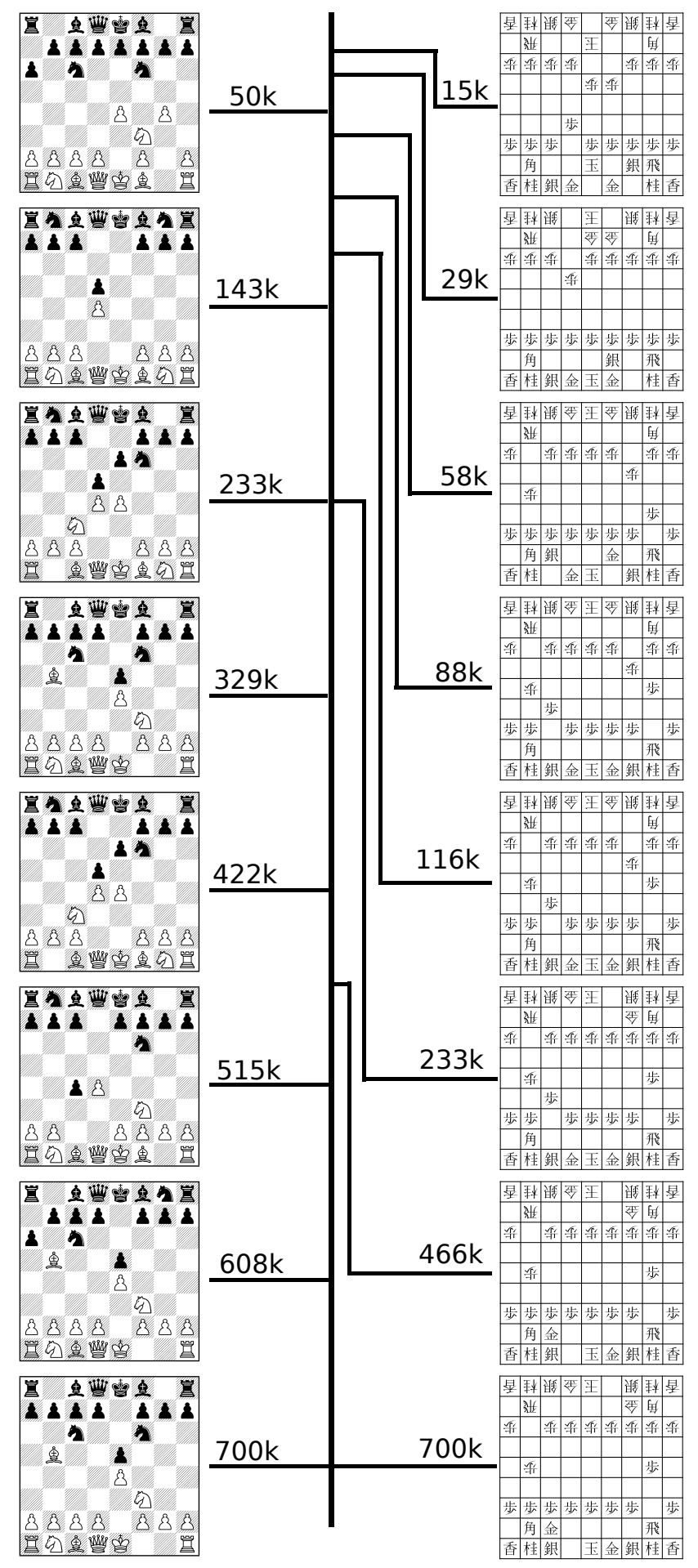

Figure S2: Chess and shogi openings preferred by AlphaZero at different stages of selfplay training, labelled with the number of training steps. The figure shows the most frequently selected opening 6 plies played by AlphaZero during its games of self-play. Each move was generated by an MCTS with just 800 simulations per move. 


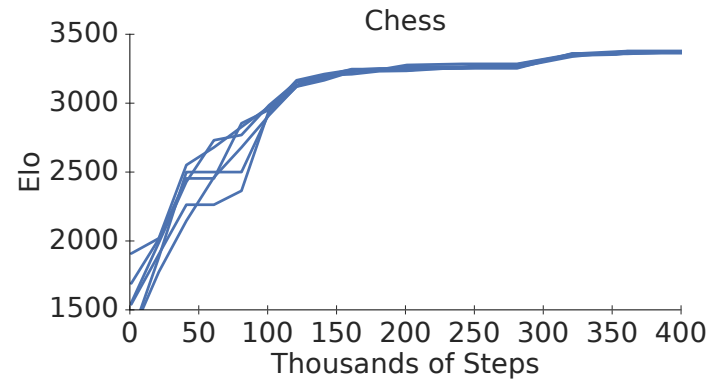

Figure S3: Repeatability of AlphaZero training on the game of chess. The figure shows 6 separate training runs of 400,000 steps (approximately 4 hours each). Elo ratings were computed from a tournament between baseline players and AlphaZero players at different stages of training. AlphaZero players were given 800 simulations per move. Similar repeatability was observed in shogi and Go. 


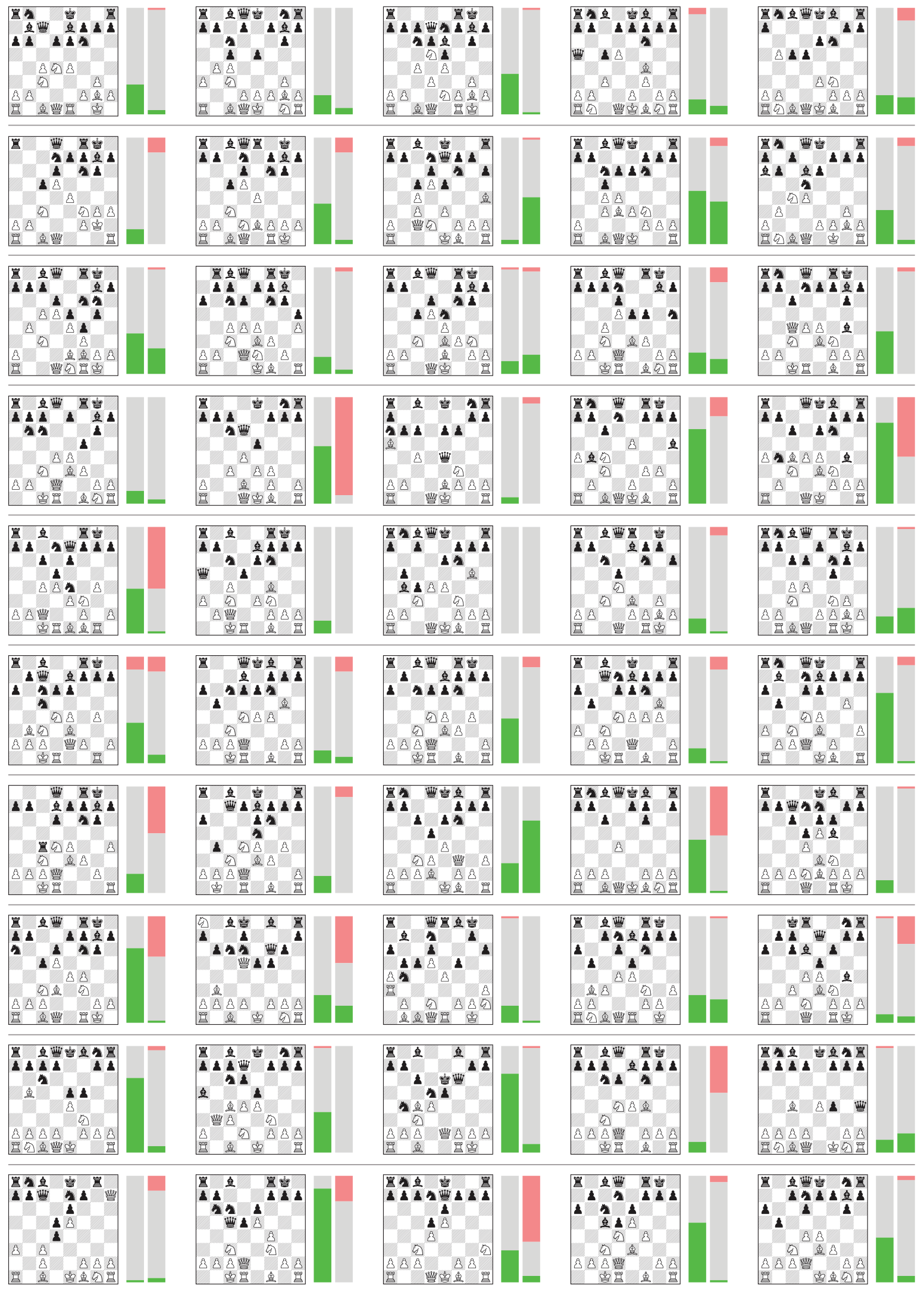

Figure S4: Chess matches beginning from the 2016 TCEC world championship start positions. In the left bar, AlphaZero plays white, starting from the given position; in the right bar AlphaZero plays black. Each bar shows the results from AlphaZero's perspective: win (green), draw (grey), loss (red). Many of these start positions are unbalanced according to both and AlphaZero and Stockfish, resulting in more losses for both players. 


\begin{tabular}{|c|c|c|c|c|c|}
\hline \multicolumn{2}{|c|}{ Go } & \multicolumn{2}{|l|}{ Chess } & \multicolumn{2}{|l|}{ Shogi } \\
\hline Feature & Planes & Feature & Planes & Feature & Planes \\
\hline P1 stone & 1 & P1 piece & 6 & P1 piece & 14 \\
\hline \multirow[t]{4}{*}{ P2 stone } & 1 & P2 piece & 6 & P2 piece & 14 \\
\hline & & Repetitions & 2 & Repetitions & 3 \\
\hline & & & & $\mathrm{P} 1$ prisoner count & 7 \\
\hline & & & & P2 prisoner count & 7 \\
\hline \multirow[t]{5}{*}{ Colour } & 1 & Colour & 1 & Colour & 1 \\
\hline & & Total move count & 1 & Total move count & 1 \\
\hline & & P1 castling & 2 & & \\
\hline & & P2 castling & 2 & & \\
\hline & & No-progress count & 1 & & \\
\hline Total & 17 & Total & 119 & Total & 362 \\
\hline
\end{tabular}

Table S1: Input features used by AlphaZero in Go, chess and shogi respectively. The first set of features are repeated for each position in a $T=8$-step history. Counts are represented by a single real-valued input; other input features are represented by a one-hot encoding using the specified number of binary input planes. The current player is denoted by P1 and the opponent by P2.

\begin{tabular}{lr|lr}
\hline & Chess & Shogi & \\
Feature & Planes & Feature & Planes \\
\hline Queen moves & 56 & Queen moves & 64 \\
Knight moves & 8 & Knight moves & 2 \\
Underpromotions & 9 & Promoting queen moves & 64 \\
& & Promoting knight moves & 2 \\
& & Drop & 7 \\
\hline Total & 73 & Total & 139 \\
\hline
\end{tabular}

Table S2: Action representation used by AlphaZero in chess and shogi respectively. The policy is represented by a stack of planes encoding a probability distribution over legal moves; planes correspond to the entries in the table. 


\begin{tabular}{llll}
\hline & Chess & Shogi & Go \\
\hline Mini-batches & $700 \mathrm{k}$ & $700 \mathrm{k}$ & $700 \mathrm{k}$ \\
Training Time & $9 \mathrm{~h}$ & $12 \mathrm{~h}$ & $13 \mathrm{~d}$ \\
Training Games & 44 million & 24 million & 140 million \\
Thinking Time & 800 sims & $800 \mathrm{sims}$ & 800 sims \\
& $\sim 40 \mathrm{~ms}$ & $\sim 80 \mathrm{~ms}$ & $\sim 200 \mathrm{~ms}$ \\
\hline
\end{tabular}

Table S3: Selected statistics of AlphaZero training in chess, shogi and Go.

\begin{tabular}{llll}
\hline Program & Chess & Shogi & Go \\
\hline AlphaZero & $63 \mathrm{k}(13 \mathrm{k})$ & $58 \mathrm{k}(12 \mathrm{k})$ & $16 \mathrm{k}(0.6 \mathrm{k})$ \\
Stockfish & $58,100 \mathrm{k}(24,000 \mathrm{k})$ & & \\
Elmo & & $25,100 \mathrm{k}(4,600 \mathrm{k})$ & \\
\hline AlphaZero & 1.5 GFlop & 1.9 GFlop & 8.5 GFlop \\
\hline
\end{tabular}

Table S4: Evaluation speed (positions/second) of AlphaZero, Stockfish, and Elmo in chess, shogi and Go. Evaluation speed is the average over entire games at full time controls from the initial board position (the main evaluation in Figure 2), standard deviations are shown in parentheses. Bottom row: Number of operations used by AlphaZero for one evaluation.

\begin{tabular}{llll}
\hline Program & Win & Draw & Loss \\
\hline Stockfish & $57.1 \%$ & $42.9 \%$ & $0.0 \%$ \\
Elmo & $98.7 \%$ & $1.0 \%$ & $0.3 \%$ \\
\hline
\end{tabular}

Table S5: Performance comparison of Stockfish and Elmo, when using full time controls of $3 \mathrm{~h}$ per game, compared to time controls of $1 \mathrm{~s}$ per move. 
Game 1 - White: AlphaZero Black: Stockfish

1. Nf3 Nf6 2. c4 e6 3. Nc3 Bb4 4. Qc2 O-O 5. a3 Bxc3 6. Qxc3 a5 7. b4 d6 8. e3 Ne4 9. Qc2 Ng5 10. b5 Nxf3+ 11. gxf3 Qf6 12 . d4 Qxf3 13. Rg1 Nd7 14. Be2 Qf6 15. Bb2 Qh4 16. Rg4 Qxh2 17. Rg3 f5 18. O-O-O Rf7 19. Bf3 Qh4 20. Rh1 Qf6 21. Kb1 g6 22. Rgg1 a4 23. Ka1 Rg7 24. e4 f4 25. c5 Qe7 26. Rc1 Nf6 27. e5 dxe5 28. Rhe1 e4 29. Bxe4 Qf8 30. d5 exd5 31. Bd3 Bg4 32. f3 Bd7 33. Qc3 Nh5 34. Re5 c6 35. Rce1 Nf6 36. Qd4 cxb5 37. Bb1 Bc6 38. Re6 Rf7 39. Rg1 Qg7 40. Qxf4 Re8 41. Rd6 Nd7 42. Qc1 Rf6 43. f4 Qe7 44. Rxf6 Nxf6 45. f5 Qe3 46. fxg6 Qxc1 47. gxh7+ Kf7 48. Rxc1 Nxh7 49. Bxh7 Re3 50. Rd1 Ke8 51. Ka2 Bd7 52. Bd4 Rh3 53. Bc2 Be6 54. Re1 Kd7 55. Kb2 Rf3 56. Re5 Rg3 57. Re3 Rg2 58. Kc3 Rg4 59. Rf3 Ke8 60. Rf2 Rg3+ 61. Kb4 Rg4 62. Rd2 Bd7 63. Ka5 Rf4 64. Be5 Rf3 65. Rd3 Rf2 66. Bd1 Bc6 67. Kb6 1-0

Game 2 - White: AlphaZero Black: Stockfish

1. d4 Nf6 2. c4 e6 3. Nf3 b6 4. g3 Bb7 5. Bg2 Be7 6. Nc3 O-O 7. O-O Ne4 8. Bd2 d5 9. cxd5 exd5 10. Qb3 c5 11. Bf4 Na6 12. Rfd1 c4 13. Qc2 Nb4 14. Qc1 Qd7 15. h4 Rac8 16. a3 Nxc3 17. bxc3 Nc6 18. Qb1 Rce8 19. Re1 Na5 20. Ng5 f5 21. Nf3 Bf6 22. Ra2 h6 23. a4 Qe6 24. Kh2 Bc8 25. Rh1 Nc6 26. h5 Kh8 27. Ng1 Qf7 28. Bf3 Rd8 29. Nh3 Kg8 30. Bc1 Rfe8 31. Qb5 Bb7 32. Rd1 Na5 33. Qb1 Bc8 34. Nf4 Bg5 35. Ng6 Bxc1 36. Qxc1 Be6 37. Ne5 Qc7 38. Rb2 Nb7 39. Rb5 Na5 40. Qf4 Nb3 41. Ng6 Qc6 42. Qe5 Qd7 43. e3 Na5 44. Bg2 Nb7 45. Ra1 Kh7 46. Nf4 Bg8 47. Rxd5 Bxd5 48. Qxd5 Nd6 49. Bh3 Re7 50. Ng6 Rf7 51. Ne5 Qb7 52. Bg2 Qxd5 53. Bxd5 Rc7 54. Kg2 Ne4 55. Bxe4 fxe4 56. f3 exf3+ 57. Kxf3 Re7 58. Ng6 Rb7 59. e4 b5 60. axb5 Rxb5 61. Nf4 Rb3 62. Ne2 Ra8 63. e5 a5 64. d5 a4 65. d6 a3 66. d7 Kg8 67. Rd1 Rbb8 68. e6 Kf8 69. Nd4 1-0

Game 3 - White: AlphaZero Black: Stockfish

1. d4 Nf6 2. c4 e6 3. Nf3 b6 4. g3 Bb7 5. Bg2 Bb4+6. Bd2 Be7 7. Nc3 O-O 8. Qc2 Na6 9. a3 c5 10. d5 exd5 11. Ng5 Nc7 12. h4 h6 13. Nxd5 Ncxd5 14. cxd5 d6 15. a4 Qd7 16. Bc3 Rfe8 17. O-O-O Bd8 18. e4 Ng4 19. Bh3 hxg5 20. f3 f5 21. fxg4 fxg4 22. Bf1 gxh4 23. Bb5 Qf7 24. gxh4 Bf6 25. Rhf1 Rf8 26. Bxf6 gxf6 27. Rf4 Qg7 28. Be2 Qh6 29. Rdf1 g3 30. Qd3 Kh8 31. Qxg3 Rae8 32. Bd3 Bc8 33. Kb1 Rf7 34. Qf2 Bd7 35. h5 Ref8 36. Bc2 Be8 37. Rf3 Re7 38. Rxf6 Qxf6 39. Qxf6+ Rxf6 40. Rxf6 Kg7 41. Rxd6 Bxh5 42. Kc1 Re5 43. a5 bxa5 44. Kd2 Be8 45. Ra6 Rh5 46. Bd3 a4 47. d6 Bf7 48. d7 Rh8 49. e5 1-0

Game 4 - White: AlphaZero Black: Stockfish

1. Nf3 e6 2. c4 Nf6 3. Nc3 Bb4 4. Qc2 O-O 5. a3 Bxc3 6. Qxc3 d6 7. b4 e5 8. Bb2 Nbd7 9. e3 Re8 10 d3 Nf8 11. Be2 a5 12. O-O Bg4 13 . h3 Bh5 14. Oc2 h6 15 . Bc3 b6 16 . b5 N6d7 17. Rad1 Nc5 18. Ba1 Bg6 19. Qb2 Na4 20. Qa2 Nc5 21. d4 exd4 22. Nxd4 Be4 23. Bf3 Bxf3 24. gxf3 Nfe6 25. Kh2 Nxd4 26. Rxd4 Kh7 27. Qc2+ g6 28. Rf4 Qe7 29. Rg1 Rg8 30. h4 h5 31. Rg5 Kh6 32. e4 Ne6 33. Rf6 Nxg5 34. hxg5+ Kh7 35. f4 Rae8 36. Qd3 Rg7 37. f3 Kg8 38. Qd4 Kf8 39. Bc3 Rg8 40. a4 Rd8 41. Kh3 Rd7 42 . f5 gxf5 43. Rxf5 Qe6 44. Kh4 Re7 45. Qd5 Rg6 46. Kxh5 Re8 47. Bf6 Qd7 48. Kg4 Rc8 49. Qc6 Qe8 50. Qxe8+ Kxe8 51. Rd5 Rxf6 52. gxf6 Kd7 53. Kf5 c6 54. bxc6+ Kxc6 55. f4 Rh8 56. e5 1-0

Game 5 - White: AlphaZero Black: Stockfish

1. d4 Nf6 2. Nf3 e6 3. c4 b6 4. g3 Bb7 5. Bg2 Bb4+ 6. Bd2 Be7 7. Nc3 c6 8. Bf4 O-O 9. e4 d5 10. e5 Ne4 11. cxd5 cxd5 12. O-O Nxc3 13. bxc3 Ba6 14. Re1 Nc6 15. h4 Rc8 16. Re3 Rc7 17. Ng5 h6 18. Nh3 Kh7 19. Rf3 Na5 20. Qc2+ Kg8 21. Re1 Kh8 22. Qd1 Nc6 23. Be3 Bc4 24. Qd2 Kh7 25. Nf4 Qe8 26. g4 Rh8 27. Nh5 Kg8 28. Rh3 Rb7 29. Bf4 Bf8 30. Qd1 Ne7 31. Bc1 b5 32. f4 Rb6 33. Ba3 Ng6 34. Bxf8 Nxf8 35. Qd2 Qc8 36. Rf3 Qd8 37. Qf2 b4 38. cxb4 Rxb4 39. f5 Qc7 40. Rd1 Qb7 41 . Rd2 Qc7 42 . Qg3 Bb5 43. Kh2 Bd7 44 . Qf2 Rb6 45. Rc2 Rc6 46. Rb2 Rb6 47. Bf1 Qb8 48. Rxb6 axb6 49. f6 g6 50. Ng3 Be8 51. Qb2 Qd8 52. h5 Nd7 53. Kg2 g5 54. Rc3 Nxf6 55. exf6 Qxf6 56. Rf3 Qd8 57. Qb4 Kg7 58. Be2 Bc6 59. Rb3 Bd7 60. Qd6 Ba4 61. Qxd8 Rxd8 62. Rxb6 Kf8 63. Kf2 Rc8 64. Ra6 Bd7 65. Ra7 Ke8 66. Bd3 Rc3 67. Ke2 Kd8 68. Kd2 Rc7 69. Rxc7 Kxc7 70. Kc3 Ba4 71. Kb4 Bd1 72. Be2 Bc2 73. Nf1 $1-0$

Game 6 - White: AlphaZero Black: Stockfish

1. Nf3 Nf6 2. c4 c5 3. Nc3 e6 4. g3 Qb6 5. d3 d5 6. Bg2 Be7 7. cxd5 exd5 8. e4 d4 9. Nd5 Nxd5 10. exd5 O-O 11. O-O Bg4 12. h3 Bxf3 13. Qxf3 Na6 14. h4 Bd6 15. h5 Qd8 16. h6 g6 17. Re1 Nc7 18. Bd2 a5 19. a4 b6 20. Re2 Re8 21. Rxe8+ Nxe8 22. Re1 Rb8 23. b3 Nc7 24. Re2 Ra8 25. Kf1 Rb8 26. Bh3 Ra8 27. Re4 Rb8 28. Re1 Ra8 29. Bc1 Rb8 30. Re2 Bf8 31. Re5 Bd6 32. Re1 Bf8 33. Bg2 Bd6 34. Bd2 Rc8 35. Bf4 Qd7 36. g4 Re8 37. Re4 Rd8 38. Bg5 Re8 39. Qf6 Bf8 40. Qc6 Qxc6 41. dxc6 Bd6 42. f4 Kf8 43. f5 gxf5 44. gxf5 Rxe4 45. Bxe4 Be5 46. f6 Kg8 47. Bf5 Ne8 48. Kf2 Bc7 49. Kf3 Bb8 50. Bf4 Bxf4 51. Kxf4 1-0

Game 7 - White: AlphaZero Black: Stockfish

1. Nf3 Nf6 2. c4 e6 3. Nc3 d5 4. d4 c6 5. Bg5 Be7 6. e3 h6 7. Bf4 O-O 8. Qc2 Nbd7 9. g4 dxc4 10. Rg1 Nd5 11. g5 Nxf4 12. gxh6 Nh5 13. hxg7 Nxg7 14. O-O-O Qa5 15. Bxc4 Qf5 16. Qe2 Qh7 17. Rg3 Kh8 18. Rdg1 Nf5 19. Qf1 Nxg3 20. Rxg3 Rg8 21. Rh3 Rg7 22. Rxh7+ Rxh7 23. Bd3 Rg7 24. Qd1 Nf6 25. Ne5 Bd7 26. Qf3 Rf8 27. Ne2 Kg8 28. a3 Be8 29. Kb1 a5 30. e4 b5 31. Bc2 b4 32. a4 Kh8 33. Ka2 Kg8 34. Ng3 Rg5 35. Qd1 Kh8 36. Bb3 Rg7 37. Qc2 Ng4 38. Nxc6 Bxc6 39. Qxc6 Rh7 40. Qc7 Bd8 41 . Qf4 Rg8 42. Bd1 Nf6 43. h4 Nd7 44. h5 Nf6 45. d5 Re8 46. d6 Rg7 47. h6 Rh7 48. e5 Nd5 49. Qd2 Rg8 50. Bb3 Bg5 51. Qd1 Nb6 52. Ne4 1-0

Game 8 - White: Stockfish Black: AlphaZero

1. e4 e5 2. Nf3 Nc6 3. Bb5 Nf6 4. O-O Nxe4 5. d4 Nd6 6. Bxc6 dxc6 7. dxe5 Nf5 8. Qxd8+ Kxd8 9. Rd1+ Ke8 10. Nc3 Be7 11. b3 Nh4 12. Nxh4 Bxh4 13. Be3 Be7 14. Ne2 h5 15. c3 h4 16. Rd2 Rh5 17. h3 a5 18. Re1 Be6 19. f4 a4 20. Nd4 Bd7 21. b4 c5 22. bxc5 Bxc5 23. Nc2 Be7 24. Rb1 b6 25. Nb4 Be6 26. Nc6 a3 27. Kh2 f6 28. Re1 f5 29. Nd4 Bd7 30. Bf2 Rd8 31. Ree2 c5 32. Nc2 g5 33. Nxa3 g4 34. Kg1 g3 35. Be3 Ra8 36. Nc4 Rh6 37. Rb2 Ra6 38. Bc1 b5 39. Ne3 Ra4 40. c4 bxc4 41. Nd5 c3 42. Nxc3 Rc4 43. Bd2 Rc6 44. Kf1 Be6 45. Rb1 Rb4

46. Ree1 Bc4+ 47. Kg1 Rc8 48. Rbc1 Bd3 49. Nd5 Rb2 50. Bc3 Rxa2 51. Ra1 Rxa1 52. Bxa1 c4 53. Nf6+ Kd8 54. Bc3 Rb8 55. Bd4 Bb4 56. Rd1 Rb5 57. Kh1 Bc5 0-1

Game 9 - White: Stockfish Black: AlphaZero

1. e4 e5 2. Nf3 Nc6 3. Bc4 Bc5 4. d3 a6 5. Ng5 Nh6 6. O-O d6 7. a4 Bg4 8. Nf3 O-O 9. h3 Bh5 10. c3 Kh8 11. Bxh6 gxh6 12. Nbd2 Ba7 13. Bd5 Ne7 14. Bxb7 Rb8 15. Bxa6 f5 16. Kh1 Ng6 17. exf5 Nf4 18. d4 Rxf5 19. Qc2 Rf8 20. Rae1 Qf6 21. Re3 Qg7 22. Rg1 Nd5 23. Bb5 Bg6 24. Qc1 Nxe3 25. fxe3 Bf7 26. Rf1 Bd5 27. Bc4 Ba8 28. a5 e4 29. Nh2 Qg5 30. b4 Qxe3 31. Ng4 Qg5 32. Qe1 h5 33. Ne3 h4 34. Be6 Bc6 35. Bc4 d5 36. b5 Bb7 37. Bb3 Rbc8 38. a6 Ba8 39. Ba4 Bb6 40. Kg1 Qg3 41. Qxg3 hxg3 42. Ra1 Rf2 43. Ndf1 Re2 44. Nf5 Rg8 45. N1xg3 Rd2 46. Rf1 Ba5 47. Rf2 Rxf2 48. Kxf2 Bxc3 49. h4 Rf8 50. Ke3 Be1 51. Ke2 Bxg3 52. Nxg3 Rg8 53. Kf2 Rg4 54. Bd1 e3+ 55. Kf3 Rxd4 56. Be2 Rb4 57. Kxe3 d4+ 58. Kd2 Rb2+ 59. Ke1 Rb3 60. Nh5 d3 61. Bd1 Rxb5 62. Nf4 Ra5 63. g3 Rxa6 64. Nxd3 Bd5 65. Kd2 Bf7 66. g4 Kg7 67. Nf2 Ra8 68. Be2 Ra4 69. Bd1 Rd4+ 70. Ke3 Rb4 71. Nd3 Rb1 72. Kd2 Rb6 73. Ke3 Re6+ 74. Kd2 Rd6 75. Kc3 Bg6 76. Nf2 Rf6 77. Nh3 Bf7 78. Be2 Re6 79. Kd2 Rb6 80. Nf2 Bd5 81. Bd3 Rb4 82. Bf5 h6 83. Ke3 Bf7 84. Nd3 Rb5 85. Bd7 Ra5 86. Bc6 Kf8 87. Be4 Ke7 88. Bf3 Kd6 89. Nf2 Bg6 90. h5 Bb1 91. Nd1 Bh7 92. Nb2 Ke7 93. Nc4 Ra6 94. Ne5 Kf6 95. Nd7+ Kg5 96. Be2 Re6+ 97. Kf2 Re7 0-1

Game 10 - White: Stockfish Black: AlphaZero

1. e4 e5 2. Nf3 Nc6 3. Bb5 Nf6 4. O-O Nxe4 5. d4 Nd6 6. Bxc6 dxc6 7. dxe5 Nf5 8. Qxd8+ Kxd8 9. Nc3 Be7 10. Rd1+ Ke8 11. Ne4 Be6 12. b3 b6 13. h3 Rd8 14. Bb2 h5 15. Rxd8+ Bxd8 16. Rd1 h4 17. Nh2 c5 18. c4 a5 19. Nc3 Nd4 20. Ng4 Rh5 21. Kf1 Bd7 22. f3 Ne6 23. Nd5 Bg5 24. Nf2 Bd8 25. Nd3 Rf5 26. Ne3 Rg5 27. Bc3 Rh5 28. Kf2 Bc8 29. Nd5 Bg5 30. f4 Bd8 31. Ne3 Bd7 32. Nd5 Bc8 33. Ne3 g6 34. Bd2 Bb7 35. Nd5 Kd7 36. Nc1 Kc8 37. Ne2 Bc6 38. Be3 Ng7 39. Nec3 Rh8 40. Ne4 a4 41. Kf3 Nf5 42. Rd2 Re8 43. Bf2 Rg8 44. b4 cxb4 45. Nxb4 Bb7 46. Nd5 Re8 47. Kg4 Nh6+ 48. Kf3 Nf5 49. Kg4 Nh6+ 50. Kf3 Re6 51. Rd3 Nf5 52. Rd2 a3 53. Kg4 Nh6+ 54. Kf3 Nf5 55. Kg4 Nh6+ 56. Kf3 Rc6 57. Ke3 Nf5+ 58. Kd3 Re6 59. Re2 Ne7 60. Ndf6 Nf5 61. Nd5 Re8 62. Kc3 b5 63. Nc5 Bc6 64. Ne4 Bb7 65. Nc5 Bc6 66. Ne4 bxc4 67. Kxc4 Bb7 68. Re1 Ba6+ 69. Kb3 Bb7 70. Kc4 Ba8 71. Bc5 Re6 72. $\mathrm{Ng} 5 \mathrm{Bxg} 573$, fxg5 Re8 74. Nf4 Ng7 75. Bxa3 Rd8 76. Re2 Rd1 77. Rf2 Ne6 78. Nxe6 Bd5+ 79. Kb5 Bxe6 80. Bc5 Rb1+ 81. Kc6 Rd1 82. Kb5 Ra1 83. a3 Re1 84. Bd4 Rb1+ 85. Kc5 Rc1+ 86. Kb5 Bd7+ 87. Kb4 Be6 88. Kb5 Rb1+ 89. Kc5 Rd1 90. a4 Rc1+ 91. Kb5 Kb7 92. Rd2 Bb3 93. Rb2 Bc4+ 94. Kb4 Be6 95. Be3 Re1 96. Bd4 Rc1 97. Kb5 Bd7+ 98. Kb4 Ka6 99. Rb3 Rc2 100. g4 Rd2 101. Kc5 Be6 102. Rf3 Kb7 103. a5 Ra2 104. Bc3 Ra3 105. Kb5 Rb3+ 106. Kc5 Ra3 107. Re3 Ra2 108. Be1 Ra1 109. Bd2 Kc8 110. Rd3 Rd1 111. Kb5 Bd7+ 112. Kb4 Be6 113. Kc5 Ra1 114. Kd4 Ra4+ 115. Kc5 Ra1 116. a6 Rxa6 117. Be1 Kb7 118. Bxh4 Ra5+ 119. Kd4 c5+ 120. Ke4 Kc6 121. Be1 Ra2 122. Rd6+ Kb5 123. Rd3 Rh2 124. Re3 Rg2 125. Kf3 Rc2 126. Rc3 Rh2 127. Kg3 Re2 128. Bf2 Kb4 129. Rc1 c4 130. Re1 Rc2 131. Be3 c3 132. h4 Ra2 133. h5 Kb3 134. h6 Ra8 135. Rc1 c2 136. h7 Rh8 137. Rh1 Kc3 138. Kf4 Kd3 139. Rh2 Kc3 140. Ke4 Kb4 141. Kd3 Bc4+ 142. Kxc2 Be6 143. Bc1 Rc8+ 144. Kd3 Rh8 145. Ke4 Ka4 146. Kf4 Kb3 147. Rh3+ Ka4 148. Bb2 Kb5 149. Ba3 1-0

Game 11 - White: AlphaZero Black: Stockfish

1. d4 \{book\} d5 \{book\} 2. c4 \{book\} c6 \{book\} 3. Nf3 \{book\} Nf6 \{book\} 4. Nc3 book e6 \{book\} 5. e3 \{book\} Nbd7 \{book\}6. Qc2 \{book\} Bd6 \{book\} 7. g4 \{book\} Bb4 \{book\} 8. Bd2 \{book\} Qe7 \{book\} 9. Rg1 \{book\} Bxc3 \{book\} 10. Bxc3 \{book\} Ne4 \{book\}11. O-O-O \{book\} O-O \{book\} 12. Be1 \{book\} b6 13. h4 Bb7 14. Ng5 Nxg5 15. hxg5 Qxg5 16. f4 Qe7 17. Kb1 c5 18. Bd3 g6 19. cxd5 cxd4 20. e4 Rac8 21. Qh2 f6 22. f5 exd5 23. fxg6 hxg6 24. Rh1 Qg7 25. Bc2 Ne5 26. Bb3 g5 27. Bg3 Nxg4 28. Qh3 Ne3 29. Rxd4 Kf7 30. Bf2 Rh8 31. Qd7+ Kg6 32. Qxg7+ Kxg7 33. Rxh8 Rxh8 34. Bxe3 Re8 35. Ra4 a6 36. Bxb6 dxe4 37. Rd4 Bc8 38. Kc1 e3 39. Rd8 Rxd8 40. Bxd8 Kg6 41. Bb6 Bb7 42. Bc2+ Kf7 43. Bxe3 Ke6 44. Kd2 Kd6 45. Bd3 1-0

Game 12 - White: AlphaZero Black: Stockfish

1. d4 \{book\} Nf6 \{book\} 2. c4 \{book\} g6 \{book\} 3. Nc3 \{book\} Bg7 \{book\} 4. e4 book d6 \{book\} 5. f3 \{book\} O-O \{book\} 6. Be3 \{book\} c5 \{book\} 7. Nge2 \{book\} Nc6 \{book\} 8. d5 \{book\} Ne5 \{book\} 9. Ng3 \{book\} e6 \{book\} 10. Be2 \{book\} exd5 \{book\} 11. cxd5 \{book\} a6 12. Qd2 b5 13. O-O Re8 14. Bh6 Bxh6 15. Qxh6 Qe7 16. Nd1 Qf8 17. Qd2 h5 18. h4 Bd7 19. Nf2 b4 20. Rfe1 Bb5 21. f4 Ned7 22. Bf3 Qh6 23. Rad1 Rab8 24. Nh3 a5 25. Ng5 a4 26. e5 dxe5 27. f5 Bc4 28. d6 Bxa2 29. Bc6 Bb3 30. Ra1 Rf8 31. Bxa4 Bxa4 32. Rxa4 Rbe8 33. Ra7 e4 34. Rc7 Re5 35. N3xe4 Nxe4 36. Rxe4 Rxe4 37. Nxe4 Qxd2 38. Nxd2 Rd8 39. fxg6 fxg6 40. Ne4 Kf8 41. Kf1 b3 42. Ke2 Re8 43. Kd3 Ne5+ 44 . Ke3 Nf7 45. d7 Re6 46. Rxc5 Ke7 47. Rd5 Ra6 48. Nc5 Ra1 49. Rd2 Rc1 50. Kd4 Kd6 51. Nxb3 Rc6 52. Na5 Rc1 53. Rf2 Nd8 54. Nc4+ Kxd7 55. Ne5+ Ke6 56. Nxg6 Nc6+ 57 . Ke3 Re1+ 58. Kd3 Rd1+ 59. Ke2 Rh1 60. g3 Rg1 61. Nf4+ Ke7 62. Nxh5 Ne5 63. Kd2 Ra1 64. Kc3 Kd6 65. Nf4 Rc1+ 66. Rc2 Rg1 67. Rg2 Rc1+ 68. Kb3 Rc8 69. Rd2+ Ke7 70. Rd5 Kf6 71. Ka2 Rg8 72. Ne2 Ke6 73. Rd4 Ra8+ 74. Kb1 Nc6 75. Rc4 Kd6 76. Re4 Rc8 77. h5 Rg8 78. Rh4 Ke7 79. h6 Kf7 80. h7 Rh8 81. Kc2 Ne7 82. Kd3 Ng6 83. Rh6 Kg7 84. Rh5 Nf8 85. b4 Nxh7 86. Nf4 Re8 87. Rf5 Nf8 88. Kd4 Re1 89. b5 Nd7 90. Kd5 1-0 
Game 13 - White: AlphaZero Black: Stockfish

1. e4 \{book\} c6 \{book\} 2. d4 \{book\} d5 \{book\} 3. e5 \{book\} Bf5 \{book\} 4. Be3 \{book\} e6 \{book\} 5. Nd2 \{book\} Nd7 \{book\}6. Ngf3 \{book\} Ne7 \{book\} 7. Be2 \{book $\}$ Qc7 \{book\} 8. O-O \{book\} f6 \{book\} 9. c3 fxe5 10. Nxe5 Nxe5 11. dxe5 Qxe5 12. Re1 Qc7 13. Bh5+ Ng6 14. g4 Bd3 15. Nb3 Bc4 16. Nd4 e5 17. b3 Ba6 18. Bf4 O-O-O 19. Bg3 Re8 20. a4 Kb8 21. b4 Bd6 22. Nf5 Bc4 23. Qd2 Ka8 24. Qg5 Re6 25. Nxd6 Rxd6 26. Bxg6 Rxg6 27. Qxe5 Qxe5 28. Rxe5 Rxg4 29. f3 Rg6 30. a5 Rf6 31. Rae1 Rff8 32. Kf2 g6 33. Re7 h5 34. Be5 Rhg8 35. Bg7 Rc8 36. Bd4 Bb5 37. Kg3 Bd3 38. Kh4 Bf5 39. Kg5 Rcd8 40. Bg7 Rc8 41. R1e2 Rcd8 42. h4 Rc8 43. a6 bxa6 44. Bd4 Kb8 45. Bxa7+ Ka8 46. Bc5 Kb8 47. Ra2 Bd3 48. Rd2 Bb1 49. Rd1 Bc2 50. Ba7+ Ka8 51. Rde1 Bd3 52. Bb6 Kb8 53. Bd4 Bf5 54. f4 Rcd8 55. Ra1 Bd3 56. Re3 Bb5 57. Rae1 Kc7 58. Kh6 Kc8 59. Re6 Ba4 60. Bc5 Kb7 61. Rxg6 Rxg6+ 62. Kxg6 d4 63. Bxd4 Bc2+ 64. Kxh5 c5 65. Bxc5 Kc8 66. Re5 Rh8+ 67. Kg4 Bd1+68. Kg3 Rh7 69. Bd4 Kd7 70. f5 Rg7+ 71. Kf2 1-0

Game 14 - White: AlphaZero Black: Stockfish

1. d4 \{book\} Nf6 \{book\} 2. c4 \{book\} e6 \{book\} 3. Nc3 \{book\} Bb4 \{book\} 4. e3 book c5 \{book\} 5. Bd3 \{book\} Nc6 \{book\} 6 . Nf3 \{book\} Bxc3+\{book\} 7. bxc3 book d6 \{book\} 8. e4 h6 9. e5 dxe5 10. Nxe5 cxd4 11. Nxc6 bxc6 12. O-O dxc3 13. Ba3 Qc7 14. Qf3 Rb8 15. Bc5 e5 16. Rfe1 Be6 17. Qg3 Nh5 18. Qe3 Nf4 19. Bc2 f6 20. h4 Qa5 21. Rab1 Kf7 22. g3 Qxa2 23. Qe4 Qxc4 24. Qxc6 Nd3 25. Rxb8 Rxb8 26. Qc7+ Kg6 27. Bxd3+ Qxd3 28. Qxb8 Bd5 29. Kh2 c2 30. Qb2 a5 31 . Qc1 a4 32. Qe3 Qxe3 33. fxe3 Kf5 34. Kg1 Ke4 35. Kf2 Be6 36. Ke2 Bg4+ 37. Kd2 Bd1 38. Rf1 f5 39. Rf2 g6 40. Ba3 Bg4 41. Kxc2 Kxe3 42. Bc5+ Ke4 43. Kd2 g5 44. Bf8 f4 45. gxf4 gxf4 46. Ke1 Be6 47. Rd2 Kf5 48. Rd8 1-0

Game 15 - White: Stockfish Black: AlphaZero

1. d4 \{book\} f5 \{book\} 2. Nf3 \{book\} Nf6 \{book\} 3. g3 \{book\} g6 \{book\} 4. Bg2 book Bg7 \{book\} 5. O-O \{book\} O-O \{book\} 6. c4 \{book\} d6 \{book\} 7. Nc3 \{book\} c6 \{book\} 8. Rb1 \{book $\}$ a 9. Qb3 Na6 10. Rd1 h6 11. Be3 Rb8 12. Rbc1 Bd7 13. c5+ Kh7 14. Na4 Nc7 15. Bd2 Be6 16. Qc2 Ncd5 17. b3 Ra8 18. Be1 Qe8 19. e3 g5 20. Nd2 Qh5 21. Bf3 g4 22. Be2 Kh8 23. Nc4 Ne4 24. h4 Ng5 25. hxg5 hxg5 26. f3 gxf3 27. Bf1 f4 28. Rd2 fxe3 29. Nxe3 Nxe3 30. Rh2 Bh3 31. Rxh3 Qxh3 32. Bxh3 Nxc2 33 . Rxc2 Bxd4+ 34 . Bf2 Bxf2+ 35. Kxf2 Kg7 36. Nb6 Rad8 37. Rc3 Rh8 38. Be6 Rh6 39. Re3 Rf8 40. Nd7 Rh2+ 41. Kf1 Re2 42. Rxe2 fxe2+ 43. Kxe2 Rh8 44. Kd3 Rh6 45. Bg4 d5 46. Ne5 e6 47. Nd7 Kf7 48. Bxf2+ 35. Kxf2 Kg7 36. Nb6 Rad8 37. Rc3 Rh8 38. Be6 Rh6 39. Re3 Rf8 40. Nd7 Rh2+ 41. Kf1 Re2 42. Rxe2 fxe2+ 43. Kxe2 Rh8 44. Kd3 Rh6 45. Bg4 d5 $46 . \mathrm{Ne} 5$ e6 $47 . \mathrm{Nd} 7$ Kf7 48.
Ke3 Rh1 49. Bf3 Re1+ 50. Kd2 Ra1 51. Bd1 Ke7 52. Ne5 Kf6 53. Ng4+ Kf5 54. Nh6+ Ke4 55. Nf7 g4 56. Nd6+ Kd4 57. a4 Ra2+ 58. Ke1 Kxc5 59. Nxb7+ Kb6 60. Nd8 Rg2 61. Nxe6 Rxg3 62. Kf2 Rc3 63. Bxg4 Rxb3 64. Bd1 Rb4 65. Kf3 Re4 0-1

Game 16 - White: Stockfish Black: AlphaZero

1. d4 \{book\} Nf6 \{book\} 2. c4 \{book\} g6 \{book\} 3. Nc3 \{book\} Bg7 \{book\} 4. e4 book d6 \{book\} 5. f3 \{book\} O-O \{book\} 6. Be3 \{book\} c5 \{book\} 7. Nge2 \{book\} Nc6 \{book\} 8. d5 \{book\} Ne5 \{book\} 9. Ng3 \{book\} e6 \{book\} 10. Be2 \{book\} exd5 \{book\} 11. cxd5 \{book\} h5 12. O-O h4 13. Nh1 h3 14. g3 Bd7 15. Rc1 b5 16. Nf2 Re8 17. Kh1 b4 18. Nb1 Neg4 19. fxg4 Nxe4 20. Nxe4 Rxe4 21. Bf4 Qe7 22. Bf3 Bxb2 23. Bxd6 Qxd6 24. Bxe4 Bxc1 25. Qxc1 Bb5 26. Re1 Re8 27. Nd2 c4 28. Nf3 c3 29. Qf4 Qc5 30. Ne5 Re7 31. Qf6 c2 32. Nxg6 fxg6 33. Qxg6+ Kf8 34. Qf6+ Kg8 35. d6 c1=Q 36. Bh7+ Rxh7 37. Qd8+ Kf7 38. Qe7+ Kg6 39. Qe6+ Kg5 40. Qg8+ Kh6 41. Qe6+ Kg7 42. Qe7+ Kg6 43. Qe6+ Kg5 44. Qg8+ Kh6 45. Qe6+ Kg7 46. Qe7+ Kg8 47. Qd8+ Be8 48. Qxe8+ Kg7 49. Qe7+ Kg6 50. Qe6+ Kg5 51. Qg8+ Kh6 52. Qe6+ Kg5 53. Qg8+ Kh6 54. Qe6+ Kg7 55. Qd7+ Kg6 56. Qe6+ Kg7 57. Qe7+ Kg8 58. Qd8+ Kg7 59. Qd7+ Kg6 60. Qe6+1/2-1/2

Game 17 - White: Stockfish Black: AlphaZero

1. e4 \{book\} c5 \{book\} 2. Nf3 \{book\} d6 \{book\} 3. d4 \{book\} cxd4 \{book\} 4. Nxd4 \{book\} Nf6 \{book\} 5. Nc3 \{book\} a6 \{book\} 6. Bg5 \{book\} Nbd7 \{book\} 7. f4 book e6 \{book\} 8. Qe2 \{book\} Be7 \{book\} 9. O-O-O \{book\} Qc7 \{book\} 10. g4 \{book\} b5 \{book\} 11. a3 \{book\} Rb8 12. Bg2 b4 13. axb4 h6 14. Bh4 Rxb4 15. Be1 Qb6 16. Bf2 Qb7 17. Rhg1 Qc7 18. Bf3 Nb6 19. Na2 Ra4 20. Kb1 e5 21. fxe5 dxe5 22. Nb3 Nc4 23. h4 h5 24. gxh5 Be6 25. Rxg7 a5 26. Be1 Rxa2 27. Kxa2 a4 28. Nc1 Na3+ 29. Ka1 Nxc2+ 30. Kb1 Nxe1 31. Qxe1 a3 32. b3 a2+ 33. Kxa2 Nd5 34. Rxd5 Bxd5 35. Kb1 Bb7 36. Rg2 Qd6 37. Qc3 Kf8 38. Nd3 Rg8 39. Rxg8+ Kxg8 40. Kc2 Bf8 41. b4 Qa6 42. Kb3 Bc6 43. Nc5 Qf1 44. Kc2 Bb5 45. Kb3 Bh6 46. Ka3 Bc1+ 47. Kb3 Bf4 48. Ka3 Bh6 49. Nb3 Bd7 50. Nc5 Bb5 51. Nb3 Bd7 52. Nc5 Bc1+ 53. Kb3 Bb5 54. Na4 Bh6 55. Qc8+ Bf8 56. Qc3 Bh6 57. Qc8+ Bf8 58. Qc3 Bd7 59. Nc5 Bb5 60. Kb2 Bh6 61. Nb3 Bf4 62. Qc8+ Kg7 63. h6+ Bxh6 64. Qg4+ Kh7 65. Qf5+ Kg8 66. Qg4+ Kh7 67. Qf5+ Kg8 68. Qg4+ Kh7 1/2-1/2

Game 18 - White: Stockfish Black: AlphaZero

1. e4 \{book\} e5 \{book\} 2. Nf3 \{book\} Nc6 \{book\} 3. Bb5 \{book\} a6 \{book\} 4. Bxc6 \{book\} dxc6 \{book\} 5. O-O \{book\} f6 \{book\}6. d4 \{book\} Bg4 \{book\} 7. c3 \{book\} Bd6 \{book\} 8. Be3 \{book\} Qe7 \{book\} 9. Nbd2 \{book\} O-O-O \{book\} 10. dxe5 fxe5 11. h3 Bd7 12. b4 Nf6 13. Qb3 g5 14. Bxg5 Rdg8 15. Kh1 Rg6 16. Rg1 Be6 17. c4 Rhg8 18. Bh4 Qf8 19. Qe3 c5 20. Rac1 b6 21. b5 Nh5 22. bxa6 Qg7 23. g3 Bxh3 24. a4 Kb8 25. a5 Bc8 26. Rg2 Ka7 27. Rh2 Bg4 28. Qd3 Qf7 29. Rc3 bxa5 30. Rb3 a4 31. Rb7+ Kxa6 32. Rb1 Qe8 33. Bg5 Nf6 34. Bxf6 Rxf6 35. Qc3 Bf8 36. Rb5 Rb6 37. Qa3 Bd7 38. Qxa4+ Kb7 39. Rxb6+ cxb6 40. Qc2 h5 41. Kg2 Bg7 42. Rh1 Qf7 43. Nh4 Bc6 44. Ndf3 Rd8 45. Nf5 Bf6 46. Ne3 Qg6 47. Nd5 Rxd5 48. cxd5 Bxd5 49. Nd2 Bc6 50. Qa2 Bg5 51. f3 Bf4 52. Nf1 b5 53. Qb2 c4 54. Kf2 Bg5 55. Qxe5 Bd8 56. Ke2 h4 57. g4 Qg5 58. Qxg5 Bxg5 59. Ne3 Kb6 60. Rd1 Kc5 61. Nd5 b4 62. f4 Bh6 63. g5 Bf8 64. f5 c3 65. f6 Be8 66. Kd3 Bb5+ 67. Ke3 Kc4 68. Nb6+ Kb3 69. g6 c2 70. Re1 Be8 71. g7 Bc5+ 72. Kd2 Bf7 73. e5 Bf2 74. Re2 Bg3 75. Re4 h3 76. e6 h2 77. Re3+ Kb2 78. Nc4+ Kb1 79. Rb3+ Ka1 80. exf7 h1=Q 81. Kxc2 Qe4+ 82. Kd2 Bf4+ 83. Ne3 Qd5+ 84. Ke2 Qh5+ 85. Kd3 Qxf7 86. Rxb4 Bg5 87. Ng4 Ka2 88. Rd4 Qe8 89. Re4 Qd7+ 90. Ke2 Qd5 91. Kf3 Ka1 92. Ne3 Qf7 93. Ng4 Qg6 94. Ra4+ Kb1 95. Ke2 Qe8+ 96. Kf2 Qe6 97. Kf1 Qf5+ 98. Ke1 Bh4+ 99. Ke2 Qc2+ 100. Kf3 Qd1+ 101. Ke3 Qd5 102. Kf4 Kb2 103. Ke3 Kc2 104. Kf4 Bg5+ 105. Kg3 Kb2 106. Kh2 Qd2+ 107. Kg1 Qd1+ 108. Kf2 Qc2+ 109. Ke1 Qg6 110. Ne5 Qe8 111. Re4 Qg8 112. Ng4 Kc3 113. Ke2 Qa2+ 114. Kf3 Qd5 115. Ne3 Qf7 116. Re7 Qxf6+ 117. Kg4 Qg6 118. Nd5+ Kd2 119. Nf4 Bxf4+ 120. Kxf4 Kd1 121. Kf3 Qg5 122. Rd7+ Kc1 123. Ra7 Kb2 124. Rb7+ Kc3 125. Ra7 Kb3 126. Rc7 Qf5+ 127. Kg3 Qe6 128. Ra7 Qg6+ 129. Kf3 Kb2 130. Rb7+ Kc2 131. Ra7 Qf5+ 132. Kg2 Qg4+ 133. Kf2 Kb3 134. Re7 Kb2 135. Re2+ Kc1 136. Re1+ Kd2 137. Re7 Qg5 138. Ra7 Kc2 139. Rc7+ Kd3 140. Kf3 Qf5+ 141. Kg3 Qg6+ 142. Kf3 Qe4+ 143. Kg3 Qe6 144. Ra7 Qg6+ 145. Kf3 Qf5+ 146. Kg3 Qg5+ 147. Kf3 Kc3 148. Rc7+ Kb3 149. Ra7 Qg6 150. Rb7+ Kc3 151. Ra7 Qf5+ 152. Ke3 Qg4 153. Re7 Kc2 154. Rb7 Qg5+ 155. Kf3 Kc3 156. Ra7 Qf5+ 157. Ke3 Qg4 158. Re7 Kc2 159. Rb7 Qe6+ 160. Kf4 Kd2 161. Rd7+ Ke2 162. Re7 Qxe7 163. g8=Q Qe3+ 164. Kf5 Qd3+ 165. Kf4 Qf3+ 166. Ke5 Qc3+ 167. Ke4 Qc2+ 168. Ke5 Qc3+ 169. Ke4 Qc2+ 170. Ke5 Qb2+ 171. Ke4 Qb1+ 172. Ke5 Qa1+ 173. Ke4 Qb1+ 174. Ke5 Qb2+ 175. Ke4 Qb4+ 176. Kf5 Qc5+ 177. Ke4 Qe7+ 178. Kf5 Qc5+179. Ke4 Qe3+ 180. Kf5 Qd3+ 181. Kf4 Qd4+ 182. Kf5 Qf2+ 183. Ke4 Qf3+184. Ke5 Qe3+185. Kf6 Qf4+ 186. Kg7 Qe5+ 187. Kg6 Qg3+ 188. Kh7 Qh4+ 189. Kg6 Qg3+ 190. Kf7 Qc7+ 191. Kf6 Qf4+ 192. Kg7 Qd4+ 193. Kg6 Qe4+ 194. Kf7 Qf5+ 195. Ke8 Qb5+ 196. Ke7 Qc5+ 197. Kf6 Qc3+ 198. Kf5 Qf3+ 199. Kg6 Qg4+ 200. Kh7 Qe4+ 201. Kh6 Qh4+ 202. Kg7 Qd4+ 203. Kg6 Qg4+ 204. Kh7 Qd7+ 205. Kg6 Qc6+ 206. Kf5 Qb5+ 207. Ke4 Qa4+ 208. Kf5 Qc2+ 209. Kf4 Qc1+210. Kf5 Qf1+ 211. Ke4 Qf3+ 212. Ke5 1/2-1/2

Game 19 - White: Stockfish Black: AlphaZero

1. e4 \{book\} e5 \{book\} 2. Nf3 \{book\} Nc6 \{book\} 3. Bb5 \{book\} f5 \{book\} 4. Nc3 fxe4 5. Nxe4 Nf6 6. Nxf6+ Qxf6 7. Qe2 Be7 8. Bxc6 bxc6 9 . Nxe5 Bb7 10. O-O O-O-O 11. d3 Rde8 12. Nc4 h5 13. Qe3 h4 14. Qh3 Kb8 15. Bd2 d5 16. Bc3 d4 17. Bd2 Bc8 18. Qf3 Qxf3 19. gxf3 Be6 20. f4 h3 21. Ne5 Bf6 22. Nxc6+ Kc8 23. Rfe1 Bd5 24. Rxe8+ Rxe8 25. Ne5 Bxe5 26. Re1 Kd7 27. fxe5 Re6 28. c4 dxc3 29. Bxc3 Rg6+ 30. Kf1 Bxa2 31. Re3 Be6 32. Rg3 Rxg3 33, fxg3 Bg4 34. e6+ Kxe6 35. Bxg7 Kf5 36. b4 c6 37. Bd4 a6 38 . Kf2 Bd1 39. Bc5 Kg4 40. Ke3 Ba4 41. Bd4 Bc2 42. Ke4 Ba4 43. Bc5 Bb5 44. Be3 Ba4 45. d4 Bd1 46. Bf4 Ba4 47. Ke5 Bb5 48. Kf6 Ba4 49. Ke6 Bb5 50. Kd6 Kh5 51. Kc5 Ba4 52. Kb6 Bb5 53. Be5 Kg5 54. Bh8 Kg6 55. Ka5 Kg5 56. Bg7 Kg6 57. Bf8 Kh5 58. Be7 Bf1 59. Kb6 Bb5 60. Bd8 Kg4 61. Bc7 Kg5 62. Kc5 Ba4 63. Kd6 Bb5 64. Bd8+ Kh5 65. Kc5 Kg4 66. Bc7 Kh5 67. Bb6 Ba4 68. Bd8 Kg4 69. Bf6 Bb5 70. Kb6 Kh5 71. Bg7 Kg5 72. Ka7 Kh5 73. Bf6 Kg4 74. Be5 Kh5 75. Kb8 Kg5 76. Kb7 Kh5 77. Ka7 Kg5 78. Bh8 Kh5 79. Kb8 Kg4 80. Kc7 Kg5 81. Kd6 Kh5 82. Bg7 Ba4 83. Bf6 Bb5 84. Ke7 Kg4 85. Bh8 Kg5 86. Ke6 Kg4 87. Be5 Ba4 88. Kf6 Bb5 89. Ke7 Kg5 90. Bc7 Kg4 91. Kd8 Kg5 92. Kc8 Kh5 93. Bd6 Kg4 94. Kb7 Kf3 95. g4 Kxg4 96. Kb6 Kf3 97. Be5 Ke4 98. Kc5 Ba4 99. Kc4 Bb5+ 100. Kc5 Ba4 101. Bg3 Bb5 102. Bd6 Ba4 103. Bc7 Bb5 104. Bd6 Ba4 105. Kc4 Bb5+ 106. Kc5 1/2-1/2

Game 20 - White: Stockfish Black: AlphaZero

1. d4 \{book\} d5 \{book\} 2. c4 \{book\} Nc6 \{book\} 3. Nf3 \{book\} Bg4 \{book\} 4. cxd5 \{book\} Bxf3 \{book\} 5 . gxf3 \{book\} Qxd5 \{book\} 6. e3 $\{$ book\} e5 $\{$ book $\} 7$. Nc3 book Bb4 \{book $\}$ 8. Bd2 \{book $\}$ Bxc3 \{book 9 9. bxc3 \{book $\}$ Qd6 \{book 10. Qb3 Nge7 11. Qxb7 O-O 12. Qa6 Rfd8 13. Rd1 Rab8 14. h4 h5 15. Be2 Rb6 16. Qc4 Rdb8 17. Qa4 Qg6 18. Kf1 Rb1 19. e4 exd4 20. cxd4 Qd6 21. d5 Ne5 22. f4 N5g6 23. Rh3 c6 24. f5 Ne5 25. Rxb1 Rxb1+ 26. Kg2 Nxf5 27. Bxh5 Qc5 28. Bd1 Rb2 29. Rc3 Nxh4+ 30. Kf1 Qb6 31. Be3 Qd8 32. Bc1 Rb6 33. Qxa7 cxd5 34. exd5 Rb5 35. d6 Qxd6 36. Bc2 g5 37. Ba3 Qd8 38. Be7 Qe8 39. Bf6 Rb8 40. a4 Ng4 41. Qe7 Nh2+ 42. Ke2 Ng4 43. Bxg5 Qxe7+ 44 . Bxe7 Re8 45. Rc7 Nf6 46. Kd3 Nd5 47. Rc4 Nf3 48. Bd6 Rd8 49. Rg4+ Kh8 50. Ke2 Nf6 51. Rc4 Ng1+ 52. Kf1 Rxd6 53. Kxg1 Ra6 54. Rc5 Nd7 55. Rd5 Nf6 56. Rf5 Rc6 57. Bd3 Rc1+ 58. Kg2 Kg7 59. a5 Rc3 60. Rf3 Rc1 61. a6 Ne8 62. Re3 Nc7 63. Bf1 Ra1 64. Re7 Nxa6 65. Ra7 Rxf1 66. Kxf1 Nc5 67. Ke2 Kg6 68. Ke3 f6 69. Rc7 Ne6 70. Rc6 Ng7 71. Kf4 Nf5 72. Ke4 Ne7 73. Rc5 Kg7 74. f4 Kf7 75. f5 Kg7 76. Kf4 Kg8 77. Kg4 1-0

Table S6: Games played by AlphaZero against Stockfish, selected by GM Matthew Sadler. The first 10 games are from the initial board position, the remaining 10 games are from 2016 TCEC world championship start positions. 
Game 1 - Black: AlphaZero White: Elmo

$+2726 \mathrm{FU}-8384 \mathrm{FU}+2625 \mathrm{FU}-8485 \mathrm{FU}+9796 \mathrm{FU}-4132 \mathrm{KI}+3938 \mathrm{GI}-9394 \mathrm{FU}+6978 \mathrm{KI}-7172 \mathrm{GI}+3736 \mathrm{FU}-3334 \mathrm{FU}+2524 \mathrm{FU}-2324 \mathrm{FU}+2824 \mathrm{HI}-8586 \mathrm{FU}+8786 \mathrm{FU}-8286 \mathrm{HI}+5968 \mathrm{OU}$ $-8685 \mathrm{HI}+7776 \mathrm{FU}-2288 \mathrm{UM}+7988 \mathrm{GI}-0033 \mathrm{KA}+2421 \mathrm{RY}-3388 \mathrm{UM}+8977 \mathrm{KE}-8877 \mathrm{UM}+6877 \mathrm{OU}-8589 \mathrm{RY}+0024 \mathrm{KE}-0085 \mathrm{KE}+7766 \mathrm{OU}-0041 \mathrm{GI}+7868 \mathrm{KI}-8979 \mathrm{RY}+6858 \mathrm{KI}-0027 \mathrm{FU}$ $+0083 \mathrm{FU}-2728 \mathrm{TO}+2432 \mathrm{NK}-3132 \mathrm{GI}+2128 \mathrm{RY}-0054 \mathrm{KE}+6656 \mathrm{OU}-7976 \mathrm{RY}+0066 \mathrm{KI}-5466 \mathrm{KE}+6766 \mathrm{FU}-8577 \mathrm{NK}+0022 \mathrm{KA}-7283 \mathrm{GI}+4746 \mathrm{FU}-8374 \mathrm{GI}+0045 \mathrm{KE}-6152 \mathrm{KI}+5647 \mathrm{OU}-$ $7767 \mathrm{NK}+0072 \mathrm{FU}-5161 \mathrm{OU}+0082 \mathrm{KA}-6172 \mathrm{OU}+8291 \mathrm{UM}-4344 \mathrm{FU}+4533 \mathrm{NK}-4445 \mathrm{FU}+0082 \mathrm{FU}-6758 \mathrm{NK}+4958 \mathrm{KI}-4546 \mathrm{FU}+4737 \mathrm{OU}-7678 \mathrm{RY}+0068 \mathrm{KE}-7869 \mathrm{RY}+0059 \mathrm{KY}-0027 \mathrm{FU}$ $+2827 \mathrm{RY}-0047 \mathrm{KI}+3847 \mathrm{GI}-4647 \mathrm{TO}+3747 \mathrm{OU}-8193 \mathrm{KE}+2725 \mathrm{RY}-0046 \mathrm{FU}+4746 \mathrm{OU}-0045 \mathrm{FU}+2545 \mathrm{RY}-0054 \mathrm{GI}+4515 \mathrm{RY}-0071 \mathrm{KI}+0084 \mathrm{KI}-6989 \mathrm{RY}+6876 \mathrm{KE}-1314 \mathrm{FU}+1525 \mathrm{RY}$ $-0024 \mathrm{FU}+2534 \mathrm{RY}-7475 \mathrm{GI}+8281 \mathrm{TO}-7584 \mathrm{GI}+8171 \mathrm{TO}-7283 \mathrm{OU}+0075 \mathrm{FU}-8475 \mathrm{GI}+0065 \mathrm{KI}-0055 \mathrm{KI}+6555 \mathrm{KI}-5455 \mathrm{GI}+4637 \mathrm{OU}-0044 \mathrm{FU}+0065 \mathrm{KI}-7576 \mathrm{GI}+6575 \mathrm{KI}-0045 \mathrm{KE}$ $+3445 \mathrm{RY}-4445 \mathrm{FU}+3332 \mathrm{NK}-5546 \mathrm{GI}+3728 \mathrm{OU}-0038 \mathrm{KI}+2838 \mathrm{OU}-0028 \mathrm{HI}+3828 \mathrm{OU}-4637 \mathrm{NG}+2818 \mathrm{OU}-3728 \mathrm{NG}+1828 \mathrm{OU}-9495 \mathrm{FU}+9182 \mathrm{UM}-8382 \mathrm{OU}+0081 \mathrm{HI}-8292 \mathrm{OU}+0091 \mathrm{KI}$ $\%$ TORYO

Game 2 - Black: AlphaZero White: Elmo

$+2726 \mathrm{FU}-8384 \mathrm{FU}+2625 \mathrm{FU}-8485 \mathrm{FU}+2524 \mathrm{FU}-2324 \mathrm{FU}+6978 \mathrm{KI}-4132 \mathrm{KI}+2824 \mathrm{HI}-0023 \mathrm{FU}+2426 \mathrm{HI}-5142 \mathrm{OU}+7776 \mathrm{FU}-3334 \mathrm{FU}+8877 \mathrm{KA}-2277 \mathrm{UM}+7877 \mathrm{KI}-6364 \mathrm{FU}+5968 \mathrm{OU}$ $-7172 \mathrm{GI}+9796 \mathrm{FU}-7374 \mathrm{FU}+2628 \mathrm{HI}-8173 \mathrm{KE}+4958 \mathrm{KI}-8281 \mathrm{HI}+7988 \mathrm{GI}-3122 \mathrm{GI}+3948 \mathrm{GI}-2233 \mathrm{GI}+4746 \mathrm{FU}-1314 \mathrm{FU}+3736 \mathrm{FU}-7263 \mathrm{GI}+4847 \mathrm{GI}-9394 \mathrm{FU}+1716 \mathrm{FU}-4252 \mathrm{OU}$ $+6766 \mathrm{FU}-6162 \mathrm{KI}+4756 \mathrm{GI}-5354 \mathrm{FU}+7778 \mathrm{KI}-8586 \mathrm{FU}+8786 \mathrm{FU}-8186 \mathrm{HI}+8887 \mathrm{GI}-8681 \mathrm{HI}+0086 \mathrm{FU}-0022 \mathrm{KA}+2937 \mathrm{KE}-3344 \mathrm{GI}+5847 \mathrm{KI}-0088 \mathrm{FU}+7888 \mathrm{KI}-4455 \mathrm{GI}+5655 \mathrm{GI}-$ $+6766 \mathrm{FU}-6162 \mathrm{KI}+4756 \mathrm{GI}-5354 \mathrm{FU}+7778 \mathrm{KI}-8586 \mathrm{FU}+8786 \mathrm{FU}-8186 \mathrm{HI}+8887 \mathrm{GI}-8681 \mathrm{HI}+0086 \mathrm{FU}-0022 \mathrm{KA}+2937 \mathrm{KE}-3344 \mathrm{GI}+5847 \mathrm{KI}-0088 \mathrm{FU}+7888 \mathrm{KI}-4455 \mathrm{GI}+5655 \mathrm{GI}-$ $+9996 \mathrm{KY}-0095 \mathrm{FU}+9695 \mathrm{KY}-9195 \mathrm{KY}+0096 \mathrm{FU}-7475 \mathrm{FU}+7675 \mathrm{FU}-0076 \mathrm{FU}+8776 \mathrm{GI}-0084 \mathrm{KE}+8887 \mathrm{KI}-8476 \mathrm{KE}+8776 \mathrm{KI}-0098 \mathrm{GI}+0079 \mathrm{KE}-9596 \mathrm{KY}+7574 \mathrm{FU}-6374 \mathrm{GI}+5554 \mathrm{FU}$ $-0075 \mathrm{FU}+7666 \mathrm{KI}-5241 \mathrm{OU}+5453 \mathrm{TO}-6253 \mathrm{KI}+4553 \mathrm{NK}-4453 \mathrm{KA}+0054 \mathrm{FU}-5344 \mathrm{KA}+0053 \mathrm{GI}-0052 \mathrm{FU}+5344 \mathrm{NG}-4344 \mathrm{FU}+0021 \mathrm{KA}-0042 \mathrm{GI}+0092 \mathrm{KA}-0024 \mathrm{KY}+0025 \mathrm{FU}-8184 \mathrm{HI}$ $+5453 \mathrm{TO}-4253 \mathrm{GI}+0054 \mathrm{FU}-5342 \mathrm{GI}+2524 \mathrm{FU}-2324 \mathrm{FU}+5453 \mathrm{TO}-4253 \mathrm{GI}+0055 \mathrm{KY}-0054 \mathrm{KE}+0023 \mathrm{FU}-7463 \mathrm{GI}+5554 \mathrm{KY}-6354 \mathrm{GI}+0055 \mathrm{FU}-5463 \mathrm{GI}+0022 \mathrm{KI}-3233 \mathrm{KI}+0043 \mathrm{KE}$ $-5362 \mathrm{GI}+2231 \mathrm{KI}-4142 \mathrm{OU}+2322 \mathrm{TO}-3343 \mathrm{KI}+2132 \mathrm{UM}-4251 \mathrm{OU}+3243 \mathrm{UM}-8486 \mathrm{HI}+0087 \mathrm{FU}-8682 \mathrm{HI}+0093 \mathrm{KI}-8292 \mathrm{HI}+9392 \mathrm{KI}-5161 \mathrm{OU}+0091 \mathrm{HI}-0071 \mathrm{KY}+9282 \mathrm{KI}-0081 \mathrm{FU}$ +9181RY -0072GI +8191RY -7576FU +6676KI -0074KA +4757KI -0081FU +0075FU $-7483 \mathrm{KA}+8272 \mathrm{KI}-8372 \mathrm{KA}+0042 \mathrm{GI}-7283 \mathrm{KA}+5554 \mathrm{FU}-6172 \mathrm{OU}+4253 \mathrm{GI}-5253 \mathrm{FU}+5453 \mathrm{TO}$ $-6253 \mathrm{GI}+4353 \mathrm{UM}-0062 \mathrm{GI}+5343 \mathrm{UM}-0052 \mathrm{KI}+0092 \mathrm{GI}-5243 \mathrm{KI}+9283 \mathrm{NG}-7261 \mathrm{OU}+0021 \mathrm{KA}-4353 \mathrm{KI}+0054 \mathrm{FU}-5352 \mathrm{KI}+9181 \mathrm{RY}-0072 \mathrm{KA}+8182 \mathrm{RY}-7283 \mathrm{KA}+8283 \mathrm{RY}-0072 \mathrm{GI}$ $+8393 \mathrm{RY}-0086 \mathrm{FU}+0091 \mathrm{KA}-8687 \mathrm{TO}+7987 \mathrm{KE}-9697 \mathrm{NY}+7868 \mathrm{OU}-9887 \mathrm{GI}+7666 \mathrm{KI}-8788 \mathrm{GI}+5747 \mathrm{KI}-8877 \mathrm{GI}+6877 \mathrm{OU}-0065 \mathrm{KE}+7768 \mathrm{OU}-0053 \mathrm{FU}+5453 \mathrm{TO}-5253 \mathrm{KI}+0054 \mathrm{FU}$ $+8393 \mathrm{RY}-0086 \mathrm{FU}+0091 \mathrm{KA}-8687 \mathrm{TO}+7987 \mathrm{KE}-9697 \mathrm{NY}+7868 \mathrm{OU}-9887 \mathrm{GI}+7666 \mathrm{KI}-8788 \mathrm{GI}+5747 \mathrm{KI}-8877 \mathrm{GI}+6877 \mathrm{OU}-0065 \mathrm{KE}+7768 \mathrm{OU}-0053 \mathrm{FU}+5453 \mathrm{TO}-5253 \mathrm{KI}+0054 \mathrm{FU}$
$-6354 \mathrm{GI}+0042 \mathrm{GI}-0057 \mathrm{FU}+3141 \mathrm{KI}-0077 \mathrm{FU}+9182 \mathrm{UM}-7778 \mathrm{TO}+6878 \mathrm{OU}-5363 \mathrm{KI}+0052 \mathrm{FU}-6152 \mathrm{OU}+2132 \mathrm{UM}-9787 \mathrm{NY}+7887 \mathrm{OU}-6577 \mathrm{NK}+8777 \mathrm{OU}-7365 \mathrm{KE}+7788 \mathrm{OU}-6577 \mathrm{NK}$ $+8877 \mathrm{OU}-0065 \mathrm{KE}+7786 \mathrm{OU}-0085 \mathrm{FU}+8696 \mathrm{OU}-0095 \mathrm{FU}+9685 \mathrm{OU}-0073 \mathrm{KE}+8594 \mathrm{OU}-3435 \mathrm{FU}+8291 \mathrm{UM} \% \mathrm{TORYO}$

Game 3 - Black: AlphaZero White: Elmo

$+6978 \mathrm{KI}-3334 \mathrm{FU}+7776 \mathrm{FU}-8384 \mathrm{FU}+2726 \mathrm{FU}-4132 \mathrm{KI}+2625 \mathrm{FU}-8485 \mathrm{FU}+2524 \mathrm{FU}-2324 \mathrm{FU}+2824 \mathrm{HI}-8586 \mathrm{FU}+8786 \mathrm{FU}-8286 \mathrm{HI}+2434 \mathrm{HI}-2233 \mathrm{KA}+5958 \mathrm{OU}-5152 \mathrm{OU}+3736 \mathrm{FU}$ $-8676 \mathrm{HI}+8877 \mathrm{KA}-0026 \mathrm{FU}+0028 \mathrm{FU}-2627 \mathrm{TO}+2827 \mathrm{FU}-3377 \mathrm{UM}+7877 \mathrm{KI}-7674 \mathrm{HI}+3474 \mathrm{HI}-7374 \mathrm{FU}+0028 \mathrm{KA}-0073 \mathrm{KA}+0083 \mathrm{HI}-7172 \mathrm{GI}+8388 \mathrm{RY}-7328 \mathrm{UM}+3928 \mathrm{GI}-0073 \mathrm{KA}$ $+0046 \mathrm{KA}-0087 \mathrm{FU}+7787 \mathrm{KI}-7346 \mathrm{KA}+4746 \mathrm{FU}-0055 \mathrm{KA}+8777 \mathrm{KI}-5546 \mathrm{KA}+4939 \mathrm{KI}-0026 \mathrm{FU}+2726 \mathrm{FU}-0027 \mathrm{FU}+2837 \mathrm{GI}-4637 \mathrm{UM}+2937 \mathrm{KE}-2728 \mathrm{TO}+3928 \mathrm{KI}-0039 \mathrm{HI}+1918 \mathrm{KY}$ $-3919 \mathrm{RY}+5847 \mathrm{OU}-1949 \mathrm{RY}+4756 \mathrm{OU}-8173 \mathrm{KE}+6766 \mathrm{FU}-0058 \mathrm{GI}+0038 \mathrm{KA}-4939 \mathrm{RY}+2829 \mathrm{KI}-3948 \mathrm{RY}+0076 \mathrm{KA}-5859 \mathrm{GI}+8848 \mathrm{RY}-5948 \mathrm{GI}+5647 \mathrm{OU}-0069 \mathrm{HI}+4748 \mathrm{OU}-6979 \mathrm{RY}$ $+0044 \mathrm{FU}-4344 \mathrm{FU}+0083 \mathrm{FU}-7989 \mathrm{RY}+8382 \mathrm{TO}-8982 \mathrm{RY}+3816 \mathrm{KA}-0034 \mathrm{FU}+0086 \mathrm{FU}-5354 \mathrm{FU}+1634 \mathrm{KA}-0043 \mathrm{GI}+3416 \mathrm{KA}-0034 \mathrm{FU}+0045 \mathrm{FU}-1314 \mathrm{FU}+4544 \mathrm{FU}-4344 \mathrm{GI}+1634 \mathrm{KA}$ $+0044 \mathrm{FU}-4344 \mathrm{FU}+0083 \mathrm{FU}-7989 \mathrm{RY}+8382 \mathrm{TO}-8982 \mathrm{RY}+3816 \mathrm{KA}-0034 \mathrm{FU}+0086 \mathrm{FU}-5354 \mathrm{FU}+1634 \mathrm{KA}-0043 \mathrm{GI}+3416 \mathrm{KA}-0034 \mathrm{FU}+0045 \mathrm{FU}-1314 \mathrm{FU}+4544 \mathrm{FU}-4344 \mathrm{GI}+1634 \mathrm{KA}$
$-0043 \mathrm{FU}+2938 \mathrm{KI}-8284 \mathrm{RY}+7667 \mathrm{KA}-8475 \mathrm{RY}+7776 \mathrm{KI}-7555 \mathrm{RY}+0047 \mathrm{FU}-4433 \mathrm{GI}+3423 \mathrm{UM}-3223 \mathrm{KI}+6723 \mathrm{UM}-0034 \mathrm{KA}+2334 \mathrm{UM}-3334 \mathrm{GI}+0067 \mathrm{KA}-0013 \mathrm{KA}+0058 \mathrm{GI}-0045 \mathrm{KE}$ $+3745 \mathrm{KE}-3445 \mathrm{GI}+0025 \mathrm{KE}-1322 \mathrm{KA}+0023 \mathrm{HI}-5262 \mathrm{OU}+2343 \mathrm{RY}-4536 \mathrm{GI}+0037 \mathrm{FU}-0042 \mathrm{FU}+4323 \mathrm{RY}-3645 \mathrm{GI}+0041 \mathrm{KI}-0034 \mathrm{KE}+3827 \mathrm{KI}-3426 \mathrm{KE}+2726 \mathrm{KI}-2244 \mathrm{KA}+0027 \mathrm{FU}$ $-0022 \mathrm{FU}+2324 \mathrm{RY}-3132 \mathrm{GI}+2635 \mathrm{KI}-4435 \mathrm{KA}+2435 \mathrm{RY}-3241 \mathrm{GI}+6745 \mathrm{KA}-0044 \mathrm{KI}+3531 \mathrm{RY}-5545 \mathrm{RY}+3141 \mathrm{RY}-0051 \mathrm{KI}+4121 \mathrm{RY}-4525 \mathrm{RY}+0036 \mathrm{GI}-2523 \mathrm{RY}+0026 \mathrm{KA}-2326 \mathrm{RY}$ $+2726 \mathrm{FU}-0024 \mathrm{KE}+3625 \mathrm{GI}-5455 \mathrm{FU}+2131 \mathrm{RY}-0054 \mathrm{KA}+0065 \mathrm{KE}-7365 \mathrm{KE}+7665 \mathrm{KI}-5465 \mathrm{KA}+6665 \mathrm{FU}-5556 \mathrm{FU}+0095 \mathrm{KA}-0073 \mathrm{KE}+0085 \mathrm{KE}-9394 \mathrm{FU}+8573 \mathrm{NK}-7273 \mathrm{GI}+0071 \mathrm{GI}$ $-6171 \mathrm{KI}+9573 \mathrm{UM}-6273 \mathrm{OU}+3151 \mathrm{RY}-5657 \mathrm{TO}+5857 \mathrm{GI}-0056 \mathrm{KE}+5756 \mathrm{GI}-0084 \mathrm{KA}+0057 \mathrm{FU}-0062 \mathrm{GI}+0075 \mathrm{KE}-0049 \mathrm{KI}+4838 \mathrm{OU}-4948 \mathrm{KI}+3848 \mathrm{OU}-2436 \mathrm{KE}+3736 \mathrm{FU}-0059 \mathrm{KA}$ $+4858 \mathrm{OU}-5948 \mathrm{UM}+5869 \mathrm{OU}-4859 \mathrm{UM}+6979 \mathrm{OU}-5969 \mathrm{UM}+7989 \mathrm{OU}-0088 \mathrm{FU}+8998 \mathrm{OU}-6987 \mathrm{UM}+9887 \mathrm{OU}-8475 \mathrm{KA}+0085 \mathrm{KE} \% \mathrm{TORYO}$

\section{Game 4 - Black: AlphaZero White: Elmo}

$+2726 \mathrm{FU}-8384 \mathrm{FU}+2625 \mathrm{FU}-8485 \mathrm{FU}+6978 \mathrm{KI}-4132 \mathrm{KI}+9796 \mathrm{FU}-7172 \mathrm{GI}+3938 \mathrm{GI}-1314 \mathrm{FU}+3736 \mathrm{FU}-8586 \mathrm{FU}+8786 \mathrm{FU}-8286 \mathrm{HI}+5958 \mathrm{OU}-5162 \mathrm{OU}+2937 \mathrm{KE}-8636 \mathrm{HI}+7776 \mathrm{FU}$ $-6271 \mathrm{OU}+8866 \mathrm{KA}-3634 \mathrm{HI}+8977 \mathrm{KE}-0083 \mathrm{FU}+7968 \mathrm{GI}-3454 \mathrm{HI}+7879 \mathrm{KI}-7182 \mathrm{OU}+2826 \mathrm{HI}-3142 \mathrm{GI}+9695 \mathrm{FU}-2213 \mathrm{KA}+2636 \mathrm{HI}-1322 \mathrm{KA}+3656 \mathrm{HI}-5474 \mathrm{HI}+1716 \mathrm{FU}-2231 \mathrm{KA}$ $+5655 \mathrm{HI}-4251 \mathrm{GI}+5575 \mathrm{HI}-7434 \mathrm{HI}+1615 \mathrm{FU}-1415 \mathrm{FU}+2524 \mathrm{FU}-3424 \mathrm{HI}+7785 \mathrm{KE}-8384 \mathrm{FU}+8593 \mathrm{NK}-8293 \mathrm{OU}+7525 \mathrm{HI}-2434 \mathrm{HI}+9594 \mathrm{FU}-9382 \mathrm{OU}+9493 \mathrm{TO}-9193 \mathrm{KY}+0094 \mathrm{FU}$ $-9394 \mathrm{KY}+9994 \mathrm{KY}-0093 \mathrm{FU}+9493 \mathrm{KY}-8293 \mathrm{OU}+0085 \mathrm{FU}-0064 \mathrm{KY}+6677 \mathrm{KA}-8485 \mathrm{FU}+0089 \mathrm{KY}-7374 \mathrm{FU}+2585 \mathrm{HI}-0084 \mathrm{FU}+8584 \mathrm{HI}-0083 \mathrm{FU}+8485 \mathrm{HI}-0094 \mathrm{FU}+0095 \mathrm{FU}-9382 \mathrm{OU}$ $+6766 \mathrm{FU}-1516 \mathrm{FU}+9594 \mathrm{FU}-1617 \mathrm{TO}+6665 \mathrm{FU}-0073 \mathrm{KE}+8595 \mathrm{HI}-7365 \mathrm{KE}+9493 \mathrm{TO}-8193 \mathrm{KE}+0094 \mathrm{FU}-6577 \mathrm{NK}+9493 \mathrm{TO}-8273 \mathrm{OU}+0065 \mathrm{FU}-7768 \mathrm{NK}+7968 \mathrm{KI}-6465 \mathrm{KY}+9565 \mathrm{HI}$ $-8384 \mathrm{FU}+6595 \mathrm{HI}-0094 \mathrm{FU}+9394 \mathrm{TO}-1727 \mathrm{TO}+9484 \mathrm{TO}-7362 \mathrm{OU}+8483 \mathrm{TO}-0084 \mathrm{FU}+8372 \mathrm{TO}-6172 \mathrm{KI}+0065 \mathrm{KE}-0094 \mathrm{FU}+9594 \mathrm{HI}-0093 \mathrm{FU}+9493 \mathrm{RY}-6252 \mathrm{OU}+0073 \mathrm{KY}-7262 \mathrm{KI}$ $+0071 \mathrm{GI}-0061 \mathrm{GI}+7162 \mathrm{NG}-6162 \mathrm{GI}+7372 \mathrm{NY}-0071 \mathrm{GI}+7271 \mathrm{NY}-6271 \mathrm{GI}+9392 \mathrm{RY}-5241 \mathrm{OU}+0045 \mathrm{KE}-0062 \mathrm{KA}+0061 \mathrm{KI}-0064 \mathrm{KY}+0072 \mathrm{GI}-7172 \mathrm{GI}+9272 \mathrm{RY}-0071 \mathrm{GI}+6171 \mathrm{KI}$ $-6465 \mathrm{KY}+0066 \mathrm{FU}-2738 \mathrm{TO}+4938 \mathrm{KI}-0026 \mathrm{KE}+3828 \mathrm{KI}-1118 \mathrm{NY}+7161 \mathrm{KI}-0052 \mathrm{GI}+0024 \mathrm{FU}-1828 \mathrm{NY}+0071 \mathrm{GI}-5261 \mathrm{GI}+7261 \mathrm{RY}-0052 \mathrm{KI}+2423 \mathrm{TO}-3223 \mathrm{KI}+0012 \mathrm{GI}-0022 \mathrm{KI}$ $+1223 \mathrm{NG}-3437 \mathrm{RY}+0032 \mathrm{FU}-3738 \mathrm{RY}+0048 \mathrm{GI}-2223 \mathrm{KI}+7162 \mathrm{NG}-0049 \mathrm{GI}+5869 \mathrm{OU}-4958 \mathrm{NG}+6858 \mathrm{KI}-3849 \mathrm{RY}+0059 \mathrm{KA}-0077 \mathrm{KE}+6978 \mathrm{OU}-4958 \mathrm{RY}+7887 \mathrm{OU}-2819 \mathrm{NY}+0068 \mathrm{KI}$ $-4132 \mathrm{OU}+6858 \mathrm{KI}-0041 \mathrm{KI}+6251 \mathrm{NG}-4151 \mathrm{KI}+6191 \mathrm{RY}-7769 \mathrm{NK}+5977 \mathrm{KA}-6979 \mathrm{NK}+0024 \mathrm{FU}-2324 \mathrm{KI}+0025 \mathrm{FU}-2434 \mathrm{KI}+0012 \mathrm{HI}-0022 \mathrm{FU}+2524 \mathrm{FU}-3424 \mathrm{KI}+1211 \mathrm{RY}-4344 \mathrm{FU}$ $+0035 \mathrm{FU}-0095 \mathrm{FU}+7795 \mathrm{KA}-0094 \mathrm{FU}+9194 \mathrm{RY}-0093 \mathrm{FU}+9493 \mathrm{RY}-7989 \mathrm{NK}+0012 \mathrm{GI}-3243 \mathrm{OU}+4533 \mathrm{NK}-2133 \mathrm{KE}+1131 \mathrm{RY}-5142 \mathrm{KI}+9584 \mathrm{KA}-2435 \mathrm{KI}+0061 \mathrm{KA}-0041 \mathrm{GI}+0051 \mathrm{GI}$ $-0081 \mathrm{KY}+0082 \mathrm{FU}-8988 \mathrm{NK}+8796 \mathrm{OU}-0083 \mathrm{FU}+5142 \mathrm{GI}-4334 \mathrm{OU}+0036 \mathrm{KI}-3536 \mathrm{KI}+6152 \mathrm{UM}-4152 \mathrm{GI}+3133 \mathrm{RY}-3425 \mathrm{OU}+0017 \mathrm{KE}-2516 \mathrm{OU}+3336 \mathrm{RY}-0094 \mathrm{KY}+0095 \mathrm{FU}-0069 \mathrm{KA}$ $+9685 \mathrm{OU}-6996 \mathrm{UM}+8596 \mathrm{OU}-0085 \mathrm{KI}+9697 \mathrm{OU}-8898 \mathrm{NK}+9787 \mathrm{OU}-9897 \mathrm{NK}+8778 \mathrm{OU}-9787 \mathrm{NK}+7879 \mathrm{OU}-8778 \mathrm{NK}+7978 \mathrm{OU}-0086 \mathrm{KE}+7888 \mathrm{OU}-8678 \mathrm{NK}+8878 \mathrm{OU}-2223 \mathrm{FU}$ $+0027 \mathrm{KI}-1615 \mathrm{OU}+3625 \mathrm{RY} \%$ TORYO

Game 5 - Black: AlphaZero White: Elmo

$+6978 \mathrm{KI}-3334 \mathrm{FU}+7776 \mathrm{FU}-8384 \mathrm{FU}+2726 \mathrm{FU}-4132 \mathrm{KI}+2625 \mathrm{FU}-8485 \mathrm{FU}+2524 \mathrm{FU}-2324 \mathrm{FU}+2824 \mathrm{HI}-8586 \mathrm{FU}+8786 \mathrm{FU}-8286 \mathrm{HI}+2434 \mathrm{HI}-2233 \mathrm{KA}+5958 \mathrm{OU}-5141 \mathrm{OU}+3436 \mathrm{HI}$ $-8684 \mathrm{HI}+3626 \mathrm{HI}-3122 \mathrm{GI}+3736 \mathrm{FU}-0086 \mathrm{FU}+8833 \mathrm{UM}-2133 \mathrm{KE}+0088 \mathrm{FU}-0025 \mathrm{FU}+2628 \mathrm{HI}-4152 \mathrm{OU}+3938 \mathrm{GI}-5262 \mathrm{OU}+2937 \mathrm{KE}-7172 \mathrm{GI}+2829 \mathrm{HI}-8434 \mathrm{HI}+0082 \mathrm{KA}-3436 \mathrm{HI}$ $+8291 \mathrm{UM}-2223 \mathrm{GI}+9192 \mathrm{UM}-2526 \mathrm{FU}+4948 \mathrm{KI}-0074 \mathrm{KA}+9282 \mathrm{UM}-3634 \mathrm{HI}+0036 \mathrm{FU}-3436 \mathrm{HI}+7877 \mathrm{KI}-3634 \mathrm{HI}+0036 \mathrm{FU}-3436 \mathrm{HI}+7786 \mathrm{KI}-3634 \mathrm{HI}+0036 \mathrm{FU}-3436 \mathrm{HI}+8675 \mathrm{KI}$ $-7483 \mathrm{KA}+0087 \mathrm{KY}-8394 \mathrm{KA}+8292 \mathrm{UM}-0084 \mathrm{FU}+9796 \mathrm{FU}-3635 \mathrm{HI}+9695 \mathrm{FU}-9483 \mathrm{KA}+9282 \mathrm{UM}-6171 \mathrm{KI}+8271 \mathrm{UM}-6271 \mathrm{OU}+8784 \mathrm{KY}-0036 \mathrm{FU}+0046 \mathrm{KI}-3637 \mathrm{TO}+3837 \mathrm{GI}-3534 \mathrm{HI}$ $+8483 \mathrm{NY}-7283 \mathrm{GI}+3726 \mathrm{GI}-7374 \mathrm{FU}+2635 \mathrm{GI}-3414 \mathrm{HI}+7565 \mathrm{KI}-0034 \mathrm{FU}+3526 \mathrm{GI}-0025 \mathrm{FU}+0024 \mathrm{FU}-2324 \mathrm{GI}+0041 \mathrm{KA}-0023 \mathrm{KA}+9594 \mathrm{FU}-9394 \mathrm{FU}+2637 \mathrm{GI}-7162 \mathrm{OU}+0092 \mathrm{FU}$ $-8173 \mathrm{KE}+6555 \mathrm{KI}-2435 \mathrm{GI}+9291 \mathrm{TO}-8372 \mathrm{GI}+9192 \mathrm{TO}-0081 \mathrm{FU}+1716 \mathrm{FU}-0054 \mathrm{KY}+5554 \mathrm{KI}-5354 \mathrm{FU}+9281 \mathrm{TO}-7281 \mathrm{GI}+7675 \mathrm{FU}-0042 \mathrm{KI}+4132 \mathrm{UM}-2332 \mathrm{KA}+7574 \mathrm{FU}-7365 \mathrm{KE}$ $+4656 \mathrm{KI}-0053 \mathrm{KE}+0036 \mathrm{FU}-5455 \mathrm{FU}+5666 \mathrm{KI}-3345 \mathrm{KE}+3635 \mathrm{FU}-4537 \mathrm{NK}+4837 \mathrm{KI}-4344 \mathrm{FU}+3746 \mathrm{KI}-5345 \mathrm{KE}+7473 \mathrm{TO}-6273 \mathrm{OU}+7968 \mathrm{GI}-4557 \mathrm{NK}+6857 \mathrm{GI}-6557 \mathrm{NK}+5857 \mathrm{OU}$ $-0056 \mathrm{GI}+4656 \mathrm{KI}-5556 \mathrm{FU}+6656 \mathrm{KI}-0065 \mathrm{GI}+0046 \mathrm{KI}-0045 \mathrm{KI}+0074 \mathrm{FU}-7362 \mathrm{OU}+0073 \mathrm{GI}-6251 \mathrm{OU}+0055 \mathrm{KY}-4555 \mathrm{KI}+5655 \mathrm{KI}-0056 \mathrm{FU}+5758 \mathrm{OU}-0053 \mathrm{KY}+0062 \mathrm{GI}-5141 \mathrm{OU}$ $+0033 \mathrm{KE}-4131 \mathrm{OU}+6253 \mathrm{GI}-4253 \mathrm{KI}+0049 \mathrm{KE}-0057 \mathrm{GI}+4957 \mathrm{KE}-5657 \mathrm{TO}+5857 \mathrm{OU}-0056 \mathrm{FU}+5748 \mathrm{OU}-0045 \mathrm{KE}+0049 \mathrm{KE}-5354 \mathrm{KI}+3321 \mathrm{NK}-3121 \mathrm{OU}+0023 \mathrm{FU}-0022 \mathrm{KE}+0033 \mathrm{KI}$ $-4557 \mathrm{NK}+4957 \mathrm{KE}-5657 \mathrm{TO}+4857 \mathrm{OU}-0056 \mathrm{FU}+4656 \mathrm{KI}-6556 \mathrm{GI}+5556 \mathrm{KI}-0075 \mathrm{KA}+0066 \mathrm{KY}-0065 \mathrm{KE}+5665 \mathrm{KI}-0056 \mathrm{FU}+5768 \mathrm{OU}-5657 \mathrm{TO}+6857 \mathrm{OU}-3223 \mathrm{KA}+0043 \mathrm{GI} \% \mathrm{TORYO}$

Game 6 - Black: AlphaZero White: Elmo

$+6978 \mathrm{KI}-3334 \mathrm{FU}+7776 \mathrm{FU}-8384 \mathrm{FU}+2726 \mathrm{FU}-4132 \mathrm{KI}+2625 \mathrm{FU}-8485 \mathrm{FU}+2524 \mathrm{FU}-2324 \mathrm{FU}+2824 \mathrm{HI}-8586 \mathrm{FU}+8786 \mathrm{FU}-8286 \mathrm{HI}+2434 \mathrm{HI}-2233 \mathrm{KA}+5958 \mathrm{OU}-3142 \mathrm{GI}+3736 \mathrm{FU}$ $-5141 \mathrm{OU}+2937 \mathrm{KE}-0022 \mathrm{FU}+3938 \mathrm{GI}-6151 \mathrm{KI}+3435 \mathrm{HI}-8682 \mathrm{HI}+3525 \mathrm{HI}-0086 \mathrm{FU}+0085 \mathrm{FU}-9394 \mathrm{FU}+8877 \mathrm{KA}-8193 \mathrm{KE}+7988 \mathrm{GI}-9385 \mathrm{KE}+7786 \mathrm{KA}-0087 \mathrm{FU}+7887 \mathrm{KI}-9495 \mathrm{FU}$ $+8675 \mathrm{KA}-7374 \mathrm{FU}+0083 \mathrm{FU}-8283 \mathrm{HI}+0084 \mathrm{FU}-8381 \mathrm{HI}+7566 \mathrm{KA}-3366 \mathrm{KA}+6766 \mathrm{FU}-8184 \mathrm{HI}+0073 \mathrm{KA}-8483 \mathrm{HI}+7391 \mathrm{UM}-0034 \mathrm{KA}+9192 \mathrm{UM}-8384 \mathrm{HI}+9293 \mathrm{UM}-8481 \mathrm{HI}+9392 \mathrm{UM}$ $-8184 \mathrm{HI}+0086 \mathrm{FU}-3489 \mathrm{UM}+4959 \mathrm{KI}-9596 \mathrm{FU}+5968 \mathrm{KI}-8597 \mathrm{NK}+9997 \mathrm{KY}-9697 \mathrm{TO}+8897 \mathrm{GI}-8998 \mathrm{UM}+9293 \mathrm{UM}-9887 \mathrm{UM}+9384 \mathrm{UM}-0083 \mathrm{FU}+8483 \mathrm{UM}-0081 \mathrm{FU}+2529 \mathrm{HI}-0044 \mathrm{KE}$ $+0025 \mathrm{KY}-8776 \mathrm{UM}+6867 \mathrm{KI}-0048 \mathrm{KI}+5868 \mathrm{OU}-4838 \mathrm{KI}+2926 \mathrm{HI}-7687 \mathrm{UM}+2522 \mathrm{NY}-0024 \mathrm{FU}+2232 \mathrm{NY}-4132 \mathrm{OU}+0088 \mathrm{KI}-8754 \mathrm{UM}+0046 \mathrm{KE}-3837 \mathrm{KI}+4654 \mathrm{KE}-5354 \mathrm{FU}+0023 \mathrm{FU}$ $-0033 \mathrm{GI}+2629 \mathrm{HI}-0055 \mathrm{KE}+6777 \mathrm{KI}-3223 \mathrm{OU}+0022 \mathrm{FU}-2322 \mathrm{OU}+6878 \mathrm{OU}-0095 \mathrm{FU}+0025 \mathrm{FU}-3738 \mathrm{KI}+2999 \mathrm{HI}-2232 \mathrm{OU}+0076 \mathrm{FU}-7475 \mathrm{FU}+7675 \mathrm{FU}-0076 \mathrm{FU}+7776 \mathrm{KI}-0096 \mathrm{KY}$ $+9796 \mathrm{GI}-9596 \mathrm{FU}+2524 \mathrm{FU}-0022 \mathrm{FU}+0026 \mathrm{KY}-0064 \mathrm{KE}+7665 \mathrm{KI}-0095 \mathrm{KY}+8394 \mathrm{UM}-0067 \mathrm{GI}+7889 \mathrm{OU}-9697 \mathrm{TO}+9495 \mathrm{UM}-9788 \mathrm{TO}+8988 \mathrm{OU}-7162 \mathrm{GI}+0079 \mathrm{KY}-6476 \mathrm{KE}+8887 \mathrm{OU}$ $-0088 \mathrm{KI}+8796 \mathrm{OU}-8899 \mathrm{KI}+0045 \mathrm{KA}-3241 \mathrm{OU}+0072 \mathrm{HI}-4253 \mathrm{GI}+7574 \mathrm{FU}-4436 \mathrm{KE}+2423 \mathrm{TO}-0094 \mathrm{FU}+9594 \mathrm{UM}-9998 \mathrm{KI}+0034 \mathrm{FU}-0097 \mathrm{HI}+9685 \mathrm{OU}-3344 \mathrm{GI}+3433 \mathrm{TO}-4433 \mathrm{GI}$ $+0095 \mathrm{FU}-3342 \mathrm{GI}+2322 \mathrm{TO}-0071 \mathrm{FU}+7292 \mathrm{RY}-8182 \mathrm{FU}+4523 \mathrm{UM}-4152 \mathrm{OU}+2232 \mathrm{TO}-5547 \mathrm{KE}+9484 \mathrm{UM}-7668 \mathrm{NK}+9282 \mathrm{RY}-0081 \mathrm{FU}+8292 \mathrm{RY}-6879 \mathrm{NK}+7473 \mathrm{TO}-0082 \mathrm{KY}+7382 \mathrm{TO}$ $-8095 \mathrm{FU}-3342 \mathrm{GI}+2322 \mathrm{TO}-007 \mathrm{FU}+7292 \mathrm{RY}-8182 \mathrm{FU}+4523 \mathrm{UM}-4152 \mathrm{OU}+2232 \mathrm{OO}-5547 \mathrm{KE}+9484 \mathrm{UM}-7668 \mathrm{NK}+9282 \mathrm{RY}-0081 \mathrm{FU}+8292 \mathrm{RY}-6879 \mathrm{NK}+7473 \mathrm{FO}-0082 \mathrm{KY}+7382 \mathrm{TO}$ $+9382 \mathrm{OU}-0022 \mathrm{FU}+2334 \mathrm{UM}-7374 \mathrm{FU}+8281 \mathrm{OU}-7775 \mathrm{RY}+0044 \mathrm{FU}-7565 \mathrm{RY}+3242 \mathrm{TO}-5342 \mathrm{GI}+4443 \mathrm{TO}-4243 \mathrm{GI}+0044 \mathrm{KI}-4334 \mathrm{GI}+0053 \mathrm{GI}-5241 \mathrm{OU}+4434 \mathrm{KI}-0042 \mathrm{FU}+0043 \mathrm{GI}$ $-6253 \mathrm{GI}+2622 \mathrm{NY}-0031 \mathrm{KY}+0032 \mathrm{FU}-0072 \mathrm{KA}+9272 \mathrm{RY}-0092 \mathrm{GI}+8191 \mathrm{OU}-8473 \mathrm{KA}+0082 \mathrm{KA}-7382 \mathrm{KA}+7182 \mathrm{NG}-5162 \mathrm{KI}+7262 \mathrm{RY}-1314 \mathrm{FU}+3231 \mathrm{TO} \% \mathrm{TORYO}$ 


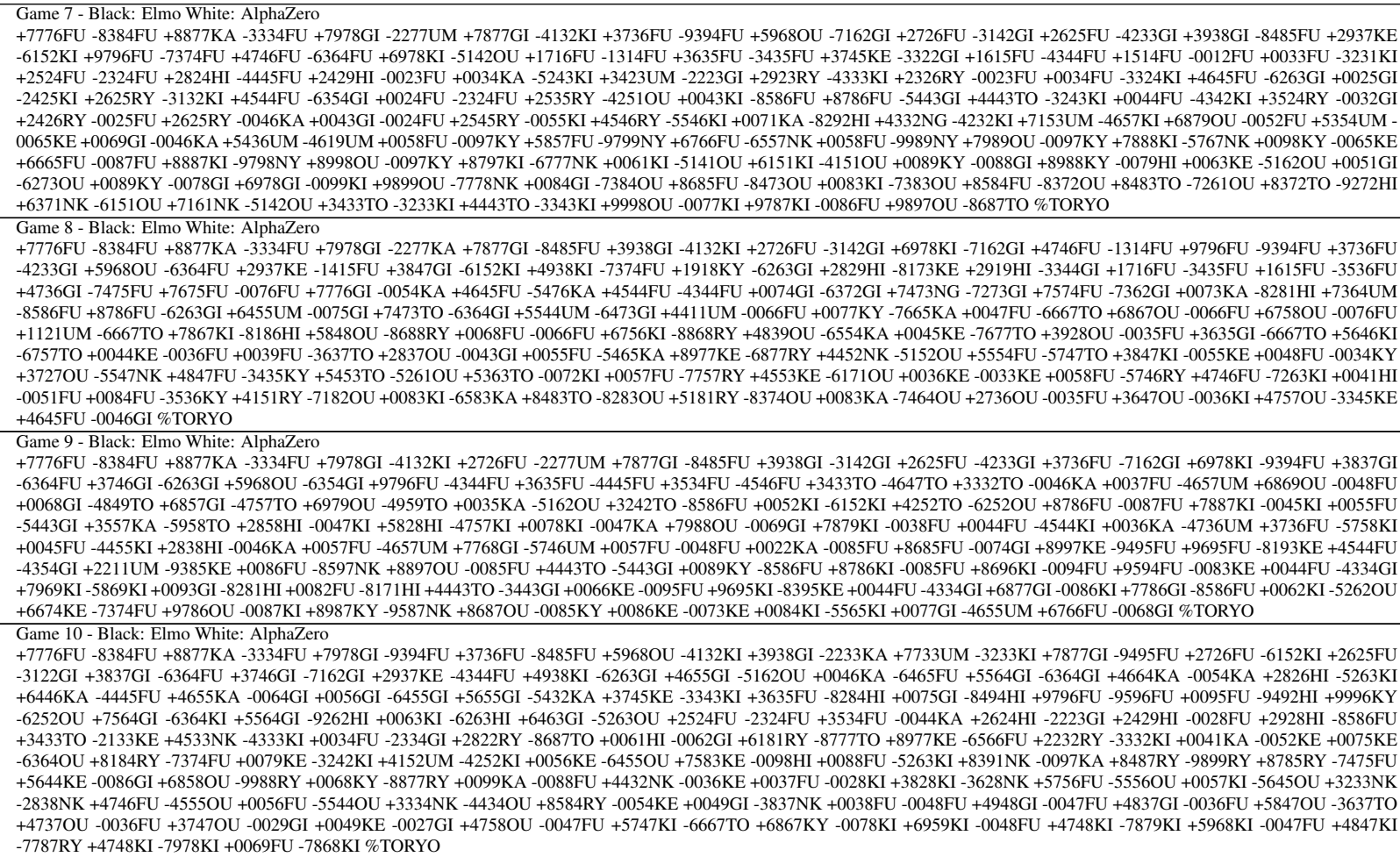

$+7776 \mathrm{FU}-8384 \mathrm{FU}+8877 \mathrm{KA}-3334 \mathrm{FU}+7978 \mathrm{GI}-4132 \mathrm{KI}+2726 \mathrm{FU}-2277 \mathrm{UM}+7877 \mathrm{GI}-8485 \mathrm{FU}+3938 \mathrm{GI}-3142 \mathrm{GI}+2625 \mathrm{FU}-4233 \mathrm{GI}+3736 \mathrm{FU}-7162 \mathrm{GI}+6978 \mathrm{KI}-9394 \mathrm{FU}+3837 \mathrm{GI}$ $-6364 \mathrm{FU}+3746 \mathrm{GI}-6263 \mathrm{GI}+5968 \mathrm{OU}-6354 \mathrm{GI}+9796 \mathrm{FU}-4344 \mathrm{FU}+3635 \mathrm{FU}-4445 \mathrm{FU}+3534 \mathrm{FU}-4546 \mathrm{FU}+3433 \mathrm{TO}-4647 \mathrm{TO}+3332 \mathrm{TO}-0046 \mathrm{KA}+0037 \mathrm{FU}-4657 \mathrm{UM}+6869 \mathrm{OU}-0048 \mathrm{FU}$ $+0068 \mathrm{GI}-4849 \mathrm{TO}+6857 \mathrm{GI}-4757 \mathrm{TO}+6979 \mathrm{OU}-4959 \mathrm{TO}+0035 \mathrm{KA}-5162 \mathrm{OU}+3242 \mathrm{TO}-8586 \mathrm{FU}+0052 \mathrm{KI}-6152 \mathrm{KI}+4252 \mathrm{TO}-6252 \mathrm{OU}+8786 \mathrm{FU}-0087 \mathrm{FU}+7887 \mathrm{KI}-0045 \mathrm{KI}+0055 \mathrm{FU}$ $-5443 \mathrm{GI}+3557 \mathrm{KA}-5958 \mathrm{TO}+2858 \mathrm{HI}-0047 \mathrm{KI}+5828 \mathrm{HI}-4757 \mathrm{KI}+0078 \mathrm{KI}-0047 \mathrm{KA}+7988 \mathrm{OU}-0069 \mathrm{GI}+7879 \mathrm{KI}-0038 \mathrm{FU}+0044 \mathrm{FU}-4544 \mathrm{KI}+0036 \mathrm{KA}-4736 \mathrm{UM}+3736 \mathrm{FU}-5758 \mathrm{KI}$ $+0045 \mathrm{FU}-4455 \mathrm{KI}+2838 \mathrm{HI}-0046 \mathrm{KA}+0057 \mathrm{FU}-4657 \mathrm{UM}+7768 \mathrm{GI}-5746 \mathrm{UM}+0057 \mathrm{FU}-0048 \mathrm{FU}+0022 \mathrm{KA}-0085 \mathrm{FU}+8685 \mathrm{FU}-0074 \mathrm{GI}+8997 \mathrm{KE}-9495 \mathrm{FU}+9695 \mathrm{FU}-8193 \mathrm{KE}+4544 \mathrm{FU}$ $-4354 \mathrm{GI}+2211 \mathrm{UM}-9385 \mathrm{KE}+0086 \mathrm{FU}-8597 \mathrm{NK}+8897 \mathrm{OU}-0085 \mathrm{FU}+4443 \mathrm{TO}-5443 \mathrm{GI}+0089 \mathrm{KY}-8586 \mathrm{FU}+8786 \mathrm{KI}-0085 \mathrm{FU}+8696 \mathrm{KI}-0094 \mathrm{FU}+9594 \mathrm{FU}-0083 \mathrm{KE}+0044 \mathrm{FU}-4334 \mathrm{GI}$ $+7969 \mathrm{KI}-5869 \mathrm{KI}+0093 \mathrm{GI}-8281 \mathrm{HI}+0082 \mathrm{FU}-8171 \mathrm{HI}+4443 \mathrm{TO}-3443 \mathrm{GI}+0066 \mathrm{KE}-0095 \mathrm{FU}+9695 \mathrm{KI}-8395 \mathrm{KE}+0044 \mathrm{FU}-4334 \mathrm{GI}+6877 \mathrm{GI}-0086 \mathrm{KI}+7786 \mathrm{GI}-8586 \mathrm{FU}+0062 \mathrm{KI}-5262 \mathrm{OU}$ +6674KE -7374FU +9786 OU $-0087 \mathrm{KI}+8987 \mathrm{KY}-9587 \mathrm{NK}+8687 \mathrm{OU}-0085 \mathrm{KY}+0086 \mathrm{KE}-0073 \mathrm{KE}+0084 \mathrm{KI}-5565 \mathrm{KI}+0077 \mathrm{GI}-4655 \mathrm{UM}+6766 \mathrm{FU}-0068 \mathrm{GI} \% \mathrm{TORYO}$

Game 10 - Black: Elmo White: AlphaZero

$+7776 \mathrm{FU}-8384 \mathrm{FU}+8877 \mathrm{KA}-3334 \mathrm{FU}+7978 \mathrm{GI}-9394 \mathrm{FU}+3736 \mathrm{FU}-8485 \mathrm{FU}+5968 \mathrm{OU}-4132 \mathrm{KI}+3938 \mathrm{GI}-2233 \mathrm{KA}+7733 \mathrm{UM}-3233 \mathrm{KI}+7877 \mathrm{GI}-9495 \mathrm{FU}+2726 \mathrm{FU}-6152 \mathrm{KI}+2625 \mathrm{FU}$

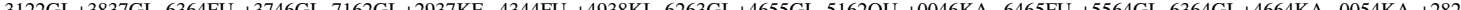
(646KA $-4445 \mathrm{FU}+4655 \mathrm{KA}-0064 \mathrm{GI}+0056 \mathrm{GI}-6455 \mathrm{GI}+565$.

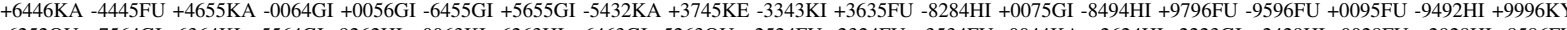

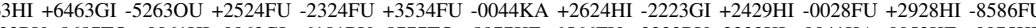
$+3433 \mathrm{TO}-2133 \mathrm{KE}+4533 \mathrm{NK}-4333 \mathrm{KI}+0034 \mathrm{FU}-2334 \mathrm{GI}+2822 \mathrm{RY}-8687 \mathrm{TO}+0061 \mathrm{HI}-0062 \mathrm{GI}+6181 \mathrm{RY}-8777 \mathrm{TO}+8977 \mathrm{KE}-6566 \mathrm{FU}+2232 \mathrm{RY}-3332 \mathrm{KI}+0041 \mathrm{KA}-0052 \mathrm{KE}+0075 \mathrm{KE}$ $-6364 \mathrm{OU}+8184 \mathrm{RY}-7374 \mathrm{FU}+0079 \mathrm{KE}-3242 \mathrm{KI}+4152 \mathrm{UM}-4252 \mathrm{KI}+0056 \mathrm{KE}-6455 \mathrm{OU}+7583 \mathrm{KE}-0098 \mathrm{HI}+0088 \mathrm{FU}-5263 \mathrm{KI}+8391 \mathrm{NK}-0097 \mathrm{KA}+8487 \mathrm{RY}-9899 \mathrm{RY}+8785 \mathrm{RY}-7475 \mathrm{FU}$ $+5644 \mathrm{KE}-0086 \mathrm{GI}+6858 \mathrm{OU}-9988 \mathrm{RY}+0068 \mathrm{KY}-8877 \mathrm{RY}+0099 \mathrm{KA}-0088 \mathrm{FU}+4432 \mathrm{NK}-0036 \mathrm{KE}+0037 \mathrm{FU}-0028 \mathrm{KI}+3828 \mathrm{KI}-3628 \mathrm{NK}+5756 \mathrm{FU}-5556 \mathrm{OU}+0057 \mathrm{KI}-5645 \mathrm{OU}+3233 \mathrm{NK}$ $-2838 \mathrm{NK}+4746 \mathrm{FU}-4555 \mathrm{OU}+0056 \mathrm{FU}-5544 \mathrm{OU}+3334 \mathrm{NK}-4434 \mathrm{OU}+8584 \mathrm{RY}-0054 \mathrm{KE}+0049 \mathrm{GI}-3837 \mathrm{NK}+0038 \mathrm{FU}-0048 \mathrm{FU}+4948 \mathrm{GI}-0047 \mathrm{FU}+4837 \mathrm{GI}-0036 \mathrm{FU}+5847 \mathrm{OU}-3637 \mathrm{TO}$ $+4737 \mathrm{OU}-0036 \mathrm{FU}+3747 \mathrm{OU}-0029 \mathrm{GI}+0049 \mathrm{KE}-0027 \mathrm{GI}+4758 \mathrm{OU}-0047 \mathrm{FU}+5747 \mathrm{KI}-6667 \mathrm{TO}+6867 \mathrm{KY}-0078 \mathrm{KI}+6959 \mathrm{KI}-0048 \mathrm{FU}+4748 \mathrm{KI}-7879 \mathrm{KI}+5968 \mathrm{KI}-0047 \mathrm{FU}+4847 \mathrm{KI}$ $-7787 \mathrm{RY}+4748 \mathrm{KI}-7978 \mathrm{KI}+0069 \mathrm{FU}-7868 \mathrm{KI} \% \mathrm{TORYO}$

Table S7: 10 games played by AlphaZero against Elmo, selected by Yoshiharu Habu. 


\begin{tabular}{|c|c|c|c|c|c|c|c|}
\hline \multirow{2}{*}{\multicolumn{2}{|c|}{ Fig. Match }} & \multirow[b]{2}{*}{ Start Position } & \multicolumn{3}{|c|}{ AlphaZero } & \multirow{2}{*}{\multicolumn{2}{|c|}{$\begin{array}{c}\text { Opponent } \\
\text { Book Main Inc Program }\end{array}$}} \\
\hline & & & Book & Main & Inc & & \\
\hline $2 \mathrm{~A}$ & Main & Initial Board & No & $3 \mathrm{~h}$ & $15 \mathrm{~s}$ & No & 3h 15 s Stockfish 8 \\
\hline $2 \mathrm{~B}$ & $1 / 100$ time & Initial Board & No & $108 \mathrm{~s}$ & $0.15 \mathrm{~s}$ & No & 3h 15 s Stockfish 8 \\
\hline $2 \mathrm{~B}$ & $1 / 30$ time & Initial Board & No & $6 \mathrm{~min}$ & $0.5 \mathrm{~s}$ & No & 3h 15 s Stockfish 8 \\
\hline $2 \mathrm{~B}$ & $1 / 10$ time & Initial Board & No & $18 \mathrm{~min}$ & $1.5 \mathrm{~s}$ & No & 3h 15 s Stockfish 8 \\
\hline $2 \mathrm{~B}$ & $1 / 3$ time & Initial Board & No & $1 \mathrm{~h}$ & $5 s$ & No & 3h 15 s Stockfish 8 \\
\hline $2 \mathrm{C}$ & latest Stockfish & Initial Board & No & $3 \mathrm{~h}$ & $15 \mathrm{~s}$ & No & 3h 15s Stockfish 2018.01.13 \\
\hline $2 \mathrm{C}$ & Opening Book & Initial Board & No & $3 \mathrm{~h}$ & $15 \mathrm{~s}$ & Yes & 3h 15 s Stockfish 8 \\
\hline $2 \mathrm{D}$ & Human Openings & Figure $3 \mathrm{~A}$ & No & $3 \mathrm{~h}$ & $15 \mathrm{~s}$ & No & 3h 15 s Stockfish 8 \\
\hline $2 \mathrm{D}$ & TCEC Openings & Figure $\overline{\mathrm{S}} 4$ & No & $3 \mathrm{~h}$ & $15 \mathrm{~s}$ & No & 3h 15 s Stockfish 8 \\
\hline
\end{tabular}

Table S8: Detailed conditions for the different evaluations in chess.

\begin{tabular}{|c|c|c|c|c|c|c|c|}
\hline \multirow[b]{2}{*}{ Fig. } & \multirow[b]{2}{*}{ Match } & \multirow[b]{2}{*}{ Start Position } & \multicolumn{3}{|c|}{ AlphaZero } & \multicolumn{2}{|r|}{ Opponent } \\
\hline & & & Book & Main & Inc & Book & Main Inc Program \\
\hline $2 \mathrm{~A}$ & Main & Initial Board & No & $3 \mathrm{~h}$ & $15 \mathrm{~s}$ & Yes & 3h $15 \mathrm{~s}$ Elmo \\
\hline $2 \mathrm{~B}$ & $1 / 100$ time & Initial Board & No & $108 \mathrm{~s}$ & $0.15 \mathrm{~s}$ & Yes & 3h $15 \mathrm{~s}$ Elmo \\
\hline $2 \mathrm{~B}$ & $1 / 30$ time & Initial Board & No & $6 \mathrm{~min}$ & $0.5 \mathrm{~s}$ & Yes & 3h $15 \mathrm{~s}$ Elmo \\
\hline $2 \mathrm{~B}$ & $1 / 10$ time & Initial Board & No & $18 \mathrm{~min}$ & $1.5 \mathrm{~s}$ & Yes & $3 \mathrm{~h} 15 \mathrm{~s}$ Elmo \\
\hline $2 \mathrm{~B}$ & $1 / 3$ time & Initial Board & No & $1 \mathrm{~h}$ & $5 \mathrm{~s}$ & Yes & $3 \mathrm{~h} 15 \mathrm{~s}$ Elmo \\
\hline $2 \mathrm{C}$ & Aperyqhapaq & Initial Board & No & $3 h$ & $15 \mathrm{~s}$ & No & 3h 15s Aperyqhapaq \\
\hline $2 \mathrm{C}$ & CSA time control & Initial Board & No & $10 \mathrm{~min}$ & $10 \mathrm{~s}$ & Yes & $10 \mathrm{~min} 10 \mathrm{~s}$ Elmo \\
\hline $2 \mathrm{D}$ & Human Openings & Figure $3 \mathrm{~B}$ & No & $3 h$ & $15 \mathrm{~s}$ & Yes & $3 \mathrm{~h} 15 \mathrm{~s}$ Elmo \\
\hline
\end{tabular}

Table S9: Detailed conditions for the different evaluations in shogi. 